\title{
Solução Numérica de Equações Integrais Singulares com Dados Não Suaves
}

\author{
Aluno: Maria Bernadete da Silva Malara \\ Orientador: Prof. Dr. José Alberto Cuminato
}

Dissertação apresentada ao Instituto de Ciências Matemáticas de São Carlos USP, como parte dos requisitos para obtenção do título de Mestre em Ciências - Área: " Ciências de Computação e Matemática Computacional ".

$$
\text { USP - São Carlos }
$$


Ao Mac, Rodrigo, Gustavo, Michel e à Adailsa 


\section{Agradecimentos}

Ao professor Dr. José Alberto Cuminato pelo incentivo, pela paciência, pela dedicação constante e orientação segura.

À professora Dra. Maria Neide Bertoldi Franco pela leitura crítica deste trabalho e sugestões oportunas, bem como, pelo estímulo e pela prontidão com que sempre me atendeu no desempenho da função de orientador substituto, tornando possivel a apresentação deste trabalho no tempo presente.

Ao Mac pela paciência, carinho e amor demonstrados durante estes anos, o que contribuiu sensivelmente para a realização deste trabalho.

Aos meus filhos Rodrigo, Gustavo e Michel pelo apoio e incentivo.

Aos meus pais, ao Ninho e à Ina pelo carinho e confiança.

À minha amiga Nadir e à tia Nailde pelo carinho e apoio emocional.

Ao Luiz Pombani pela amizade e apoio à minha família nos momentos difíceis.

Aos meus afilhados Fábio, Marcos e Milene pelo carinho.

Ao professor doutor Carlos Biasi pelo incentivo e sugestões.

À minha amiga Adailsa que me incentivou a realizar o sonho de fazer este mestrado.

À Beth e à Laura pelo apoio e pela atenção que sempre me dedicaram durante estes anos.

Aos colegas, professores e funcionários do ICMSC-USP, e a todos os que direta ou indiretamente participaram deste trabalho.

À amiga Josie que desempenhando as tarefas domésticas com muita responsabilidade deu-me o tempo de que eu necessitava para me dedicar a esta dissertação.

Ao CNPq, FAPESP e FINEP pelo constante apoio aos programas de pósgraduação, em especial à CAPES que outorgou à autora deste trabalho, bolsa de mestrado.

À Deus, pois sem Ele nada seria possível. 


\begin{abstract}
This work is concerned with the numerical solution of a constant coefficient singular integral equations having the Heaviside function as the right-hand side or forcing term by polynomial collocation method. This method uses the zeros of a Chebyshev polynomial of first kind as collocation points.
\end{abstract}




\section{RESUMO}

Este trabalho está relacionado com a solução numérica da equação integral singular com coeficientes constantes, tendo do lado direito a função de Heaviside, pelo método de colocação polinomial. Este método usa os zeros do polinômio de Chebyshev de primeira espécie como pontos de colocação. 


\section{Prefácio}

A teoria de Equações Integrais Singulares (EIS) tem se tornado um importante campo de estudo devido à sua frequência em muitos ramos da Física.

Sabe-se que através do uso de técnicas especiais, muitos problemas práticos transformam-se em EIS.

Como exemplo, temos as seguintes referências: em Aerodinâmica ([Golberg 79] e [ Bland 70]); Mecânica de Fraturas ([Erdogan 71a], [Erdogan 71b], [Erdogan 73] e [ Boiko 84]); Acústica ([Miller 82] e [Wickhan 82]); Teoria da Elasticidade ([Muskhelishvili 53]) e Hidrodinâmica ( [Zabreyko 75]).

A finalidade deste trabalho é estudar a solução numérica de EIS com coeficientes constantes e dados não suaves, com ênfase naquelas que apresentem do lado direito a função de Heaviside.

Os assuntos constantes desta dissertação, distribuídos como veremos a seguir, foram escolhidos de acordo com o seguinte princípio: "É no conhecimento teórico do comportamento da solução exata que repousam as bases de todo método numérico ".

No Capítulo 1, apresentamos os pre-requisitos, no Capítulo 2, a teoria geral de EIS e no Capítulo 3, a teoria básica de EIS com coeficientes constantes e o método de colocação polinomial para esta classe de equações.

No Capítulo 4, descrevemos uma regra de quadratura que aproxima integrais singulares com núcleo de Cauchy. Esta regra de quadratura, desenvolvida inicialmente por Apostolos Gerasoulis, recebeu uma valiosa contribuição no trabalho de Cuminato. Nesta dissertação fizemos um estudo complementar e algumas demonstrações adicionais. $\mathrm{Na}$ última seção deste capítulo desenvolvemos a fórmula de quadratura para $S_{i}(x)$, apoiada nos trabalhos de Miller e Keer.

No Capítulo 5, apresentamos a teoria de EIS com coeficientes constantes e dados não suaves e sua solução numérica, explorando a idéia de um método que associa as regras de quadraturas vistas no cápítulo anterior ao método de colocação polinomial, utilizandose de uma estratégia adequada que possibilite a aplicação deste último. 
Desenvolvemos a avaliação numérica de $K u(x)$, estudando minuciosamente os casos e modos de aplicação. Apresentamos o método de colocação polinomial para esta classe de equações. Em exemplos numéricos, propomos um algoritmo e os exemplos propriamente ditos.

Finalmente, aduzimos as referências bibliográficas usadas na elaboração deste trabalho. 


\section{Conteúdo}

1 Pré-requisitos 1

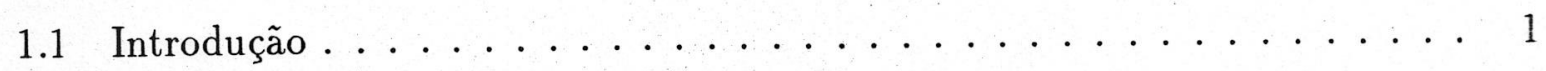

1.2 Definições, Lemas e Teoremas Básicos . . . . . . . . . . . . 1

1.3 O Problema de Hilbert para Arcos Abertos . . . . . . . . . . .

1.3.1 Solução do Problema Homogêneo . . . . . . . . . . . . 7

1.3.2 Solução do Problema não Homogêneo . . . . . . . . . . . 9

1.4 Polinômios Ortogonais de Jacobi . . . . . . . . . . . . . 9

1.5 A Função Gama e os números binomiais . . . . . . . . . . . 10

1.6 Quadratura de Gauss ....................... 11

2 Equações Integrais Singulares (EIS) com núcleo de Cauchy 12

2.1 Introdução . . . . . . . . . . . . . . 12

2.2 Equação Integral Singular . . . . . . . . . . . . . 12

2.2.1 Solução da Equação Dominante . . . . . . . . . . . 13

2.2 .2 Solução da Equação Completa . . . . . . . . . . . 16

3 Equação integral singular com coeficientes constantes 19

3.1 Introdução . . . . . . . . . . . . . . . . 19

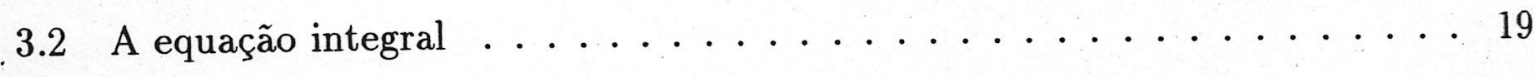

3.3 Método de Colocação Polinomial . . . . . . . . . . . 23

3.3.1 Interpolação polinomial e operador projeção . . . . . . 25 
3.3.2 Teorema de Convergência ................ 27

4 Fórmulas de Quadratura 28

4.1 Introdução . . . . . . . . . . . . . . . . . 28

4.2 A Fórmula de Gerasoulis . . . . . . . . . . . . . . . . 28

4.2 .1 Cálculo de $I_{i} \ldots \ldots \ldots \ldots \ldots$

4.2 .2 A quadratura de Gerasoulis . . . . . . . . . . . 33

4.3 Aplicações numéricas . . . . . . . . . . . . . . . . 42

4.4 Algoritmo do Ponto Médio (A.P.M.) . . . . . . . . . 43

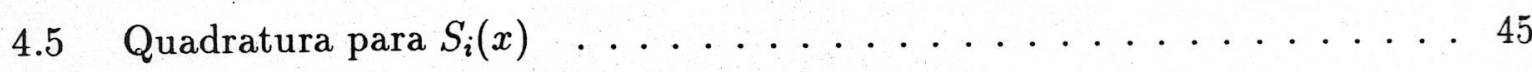

5 Equação Integral Singular com Coeficientes Constantes e Dados não $\begin{array}{ll}\text { Suaves } & 48\end{array}$

5.1 Introdução . . . . . . . . . . . . . . . . 48

5.2 Comportamento da Solução Exata da Equação Integral . . . . . . . . . . 49

5.3 Solução Numérica da Equação Integral . . . . . . . . . . . . 50

5.3 .1 Avaliação numérica da função $u \ldots \ldots \ldots \ldots$

5.3 .2 Avaliação numérica da função $K u \ldots \ldots \ldots \ldots \ldots \ldots \ldots$

5.3 .3 O método de colocação polinomial . . . . . . . . 57

5.4 Exemplos Numéricos ........................ 58

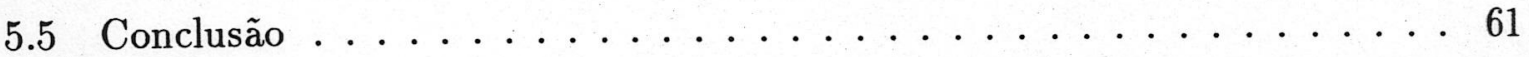




\section{Lista de Tabelas}

4.1 Número de termos da quadratura de Gerasoulis . . . . . . . . . . . . 42

4.2 Número de termos usando A.P.M. . . . . . . . . . . . . . . 44

4.3 Valor da integral usando o A.P.M. . . . . . . . . . . . 44

5.1 Solução da Equação integral do exemplo 1. . . . . . . . . . . . 60

5.2 Solução da Equação integral do Exemplo $2 . \ldots \ldots \ldots$. . . . . . 61 


\section{Capítulo 1}

\section{Pré-requisitos}

\subsection{Introdução}

Este capítulo, é todo dedicado à apresentação de resultados necessários ao desenvolvimento deste trabalho.

Inicialmente introduzimos as integrais de Cauchy e algumas de suas propriedades, criando condições para a demonstração da fórmula de Plemelj-Sokhotsky.

Mostramos, a seguir, uma síntese do Problema de Hilbert, que tem sua importância no estudo das. Equações Integrais Singulares (EIS); definimos os polinômios ortogonais de Jacobi e a maneira de obtê-los através da fórmula de recorrência. Ressaltamos os zeros dos polinômios de Chebyschev de primeira espécie, usados neste trabalho como pontos de colocação.

Definimos ainda a função Gama cujas propriedades serão usadas na demonstração da quadratura de Gerasoulis.

Finalmente, apresentamos quadratura de Gauss.

Os assuntos expostos aqui podem ser encontrados em [Cuminato 87] ou, quando for o caso, nas referências citadas.

\subsection{Definições, Lemas e Teoremas Básicos}

Definição 1.1 Contorno Suave é uma linha aberta ou fechada do plano complexo, que não se intercepta e tem tangente variando continuamente. 
Denotaremos tal contorno pela letra $\mathrm{L}$ e um número complexo genérico em $C \backslash L$ pela letra $z$. As letras $x, t, s$ serão usadas para denotar um ponto qualquer do contorno L.

No decorrer deste estudo, usamos arco aberto para designar um contorno suave aberto.

Definição 1.2 Seja L um contorno suave fechado orientado numa direção que chamaremos direção positiva. A parte do plano situada à esquerda de $L$ será sempre denotada por $D^{+} e$ à direita por $D^{-}$.

No caso em que L é um arco aberto, o conjunto

$$
S=\{z \in C / z \notin L\}
$$

será chamado plano complexo deletado.

Definição 1.3 Seja $L$ um contorno suave e $\phi(x)$ uma função definida em L. Dizemos que $\phi(x)$ satisfaz a condição de Hölder em $L$, se para pontos arbitrários $x_{1}$ e $x_{2}$ de $L$,

$$
\left|\phi\left(x_{1}\right)-\phi\left(x_{2}\right)\right| \leq A\left|x_{1}-x_{2}\right|^{\mu}
$$

onde A é uma constante positiva e $0<\mu \leq 1$.

Notação : $\phi \in H^{\mu}(L)$.

Definição 1.4 Seja $L$ um contorno suave e $\psi(x, t)$ uma função de duas variáveis em $L$. Dizemos que $\psi(x, t)$ satisfaz a condição de Hölder em $L$ se, para quaisquer pares de pontos $\left(x_{1}, t_{1}\right)$ e $\left(x_{2}, t_{2}\right)$ de $L$

$$
\left|\psi\left(x_{1}, t_{1}\right)-\psi\left(x_{2}, t_{2}\right)\right| \leq D\left[\left|x_{1}-x_{2}\right|^{\mu}+\left|t_{1}-t_{2}\right|^{\mu}\right],
$$

onde $D$ é uma constante $e 0<\mu \leq 1$.

A notação é a mesma que está sendo usada para o caso de uma variável, isto é, $\psi \in H^{\mu}(L)$.

Definição 1.5 Seja $L$ um arco aberto e $\phi(t)$ uma função definida em $L$ satisfazendo a condição de Hölder em toda parte fechada de L que não contenha os extremos. Suponhamos que nas vizinhanças de um extremo $c$ de $L$, $\phi$ seja da forma

$$
\phi(t)=\frac{\phi^{*}(t)}{(t-c)^{\alpha}} ; \quad 0<\alpha<1
$$

$\operatorname{com} \phi^{*} \in H^{\mu}(L)$.

Dizemos então que $\phi \in H^{*}$. 
Definição 1.6 [Gakhov 66] Seja $L$ um contorno suave inteiramente contido na parte finita do plano. Seja $t \in L$ e $z$ qualquer ponto do plano. Seja ainda $\phi(t)$ uma função limitada em $L$ com possivel exceção de um número finito de pontos $c_{k}$ onde, entretanto,

$$
|\phi(t)| \leq \frac{C}{|t-c|^{\alpha}}
$$

com $C$ e $\alpha$ constantes positivas, $\alpha<1$ e c representando qualquer dos pontos $c_{k}$.

Então a integral

$$
\Phi(z)=\frac{1}{2 \pi i} \int_{L} \frac{\phi(t)}{t-z} d t
$$

é chamada integral de Cauchy com densidade $\phi(t)$.

Esta função $\Phi(z)$ está bem definida no caso em que $z \notin L$ e é holomorfa no plano complexo deletado.

Definição 1.7 Seja $L$ um contorno suave e $\phi \in H^{\mu}(L)$. Define-se e denota-se o valor principal de Cauchy da integral de $\Phi$ num ponto $x$ de L , pela expressão:

$$
f_{L} \frac{\phi(t)}{t-x} d t=\lim _{\varepsilon \rightarrow 0} \int_{L-l} \frac{\phi(t)}{t-x} d t
$$

onde $L-l$ é o contorno obtido subtraindo-se de $L$, a intersecção de $L$ com um círculo centrado no ponto $x$ e raio $\varepsilon$.

Em [Muskhelishvili 53] pode ser visto que:

1. Se a integral

$$
f_{L} \frac{\phi(t)}{t-x} d t
$$

existe no sentido de Riemann então o valor principal de Cauchy também existe e eles coincidem.

2. Se a função $\phi(t)$ satisfaz a condição de Hölder na vizinhança do ponto $x$ então o valor principal sempre existe.

Definição 1.8 Seja $L$ um contorno suave e $\phi(t) \in H^{\mu}(L)$. Para cada $z \notin L$ e cada $x \in L, x$ diferente dos extremos de $L$, define-se e denota-se por :

$$
\begin{aligned}
& \Phi^{+}(x)=\lim _{z \rightarrow x} \Phi(z) ; \quad z \in D^{+}, \\
& \Phi^{-}(x)=\lim _{z \rightarrow x} \Phi(z) ; \quad z \in D^{-},
\end{aligned}
$$

onde $\Phi(z)$ é dada por (1.1). 
Lema 1.1 Seja L um contorno suave. Se a função densidade $\phi(t)$ satisfaz a condição de Hölder em $L$ e o ponto $x$ não é um extremo de $L$, então a função:

$$
\psi(z)=\frac{1}{2 \pi i} \int_{L} \frac{\phi(t)-\phi(x)}{t-z} d t
$$

é contínua em $L$, isto é,

$$
\lim _{z \rightarrow x} \psi(z)=\frac{1}{2 \pi i} \int_{L} \frac{\phi(t)-\phi(x)}{t-x} d t=\psi(x)
$$

Provar este lema consiste em estimar a diferença $|\psi(z)-\psi(x)|$, observando que $\psi(z)$ tende uniformemente, com respeito à posição de $x$ em $L$ para o limite $\psi(x)$. Esta prova pode ser encontrada em [Muskhelishvili 53].

Teorema 1.1 Seja L um contorno suave e $\Phi(z)$ a função dada por (1.1) onde a densidade $\phi(t)$ satisfaz a condição de Hölder em $L$. Então $\Phi(z)$ é contínua à esquerda ou à direita de $L$ com exceção daqueles extremos nos quais $\phi(t) \neq 0$.

A prova deste teorema, que pode ser vista em [Muskhelishvili 53], assume que $L$ é um contorno fechado, mas pode ser estendida para arcos abertos. Neste caso precisamos somente exigir que $\phi \in H^{*}$ e o teorema continua válido exceto naqueles extremos de $L$ onde $\phi(t) \neq 0$.

Teorema 1.2 [Fórmulas de Plemelj - Sokhotsky]

Seja L um contorno suave, $\Phi(z)$ como em (1.1), $\Phi^{+}(x)$ como em (1.2) e $\Phi^{-}(x)$ como em (1.3).

Se a função $\phi(t)$ satisfaz a condição de Hölder em L, então existem os limites $\Phi^{+}(x)$ e $\Phi^{-}(x)$ em todos os pontos do contorno $L$, que não coincidem com os extremos.

Além disto, se $z$ tende para $x \in L$ pela esquerda ou pela direita ao longo de um caminho arbitrário, estes valores limites são dados por:

$$
\begin{gathered}
\Phi^{+}(x)=\frac{1}{2} \phi(x)+\frac{1}{2 \pi i} f_{L} \frac{\phi(t)}{t-x} d t \\
\Phi^{-}(x)=-\frac{1}{2} \phi(x)+\frac{1}{2 \pi i} f_{L} \frac{\phi(t)}{t-x} d t
\end{gathered}
$$


Prova: Seja $L$ um contorno fechado e sejam $D^{+}$e $D^{-}$como na definição (1.2).

Do Teorema de Cauchy, temos

$$
\int_{L} \frac{1}{t-z} d t= \begin{cases}2 \pi i & z \in D^{+} \\ 0 & z \in D^{-} \\ \pi i & z \in L\end{cases}
$$

e então para $x \in L$

$$
\begin{aligned}
\Phi(z) & =\frac{1}{2 \pi i} \int_{L} \frac{\phi(t)}{t-z} d t \\
& =\frac{1}{2 \pi i} \int_{L} \frac{\phi(t)-\phi(x)}{t-z} d t+\frac{\phi(x)}{2 \pi i} \int_{L} \frac{1}{t-z} d t
\end{aligned}
$$

Do teorema 1.1 e das equações (1.6), temos

$$
\begin{aligned}
\Phi^{+}(x) & =\frac{1}{2 \pi i} \int_{L} \frac{\phi(t)-\phi(x)}{t-x} d t+\phi(x) \\
& =\frac{1}{2 \pi i}\left[f_{L} \frac{\phi(t)}{t-x} d t-\phi(x) f_{L} \frac{1}{t-x} d t\right]+\phi(x) \\
& =\frac{1}{2} \phi(x)+\frac{1}{2 \pi i} f_{L} \frac{\phi(t)}{t-x} d t
\end{aligned}
$$

A fórmula para $\Phi^{-}(x)$ demonstra-se de maneira análoga.

Adicionando e subtraindo (1.4) e (1.5) obtemos duas fórmulas equivalentes muito usadas:

$$
\begin{gathered}
\Phi^{+}(x)+\Phi^{-}(x)=\frac{1}{\pi i} f_{L} \frac{\phi(t)}{t-x} d t \\
\Phi^{+}(x)-\Phi^{-}(x)=\phi(x) .
\end{gathered}
$$

No caso em que $L$ é um arco aberto, o teorema 1.2 segue da validade do teorema 1.1 para arcos abertos assumindo-se que $\phi \in H^{*}$. A fórmula (1.8) foi obtida exigindo-se que $\phi(t)$ fosse Hölder contínua pelo menos na vizinhança de $x$. No caso em que $\phi(t)$ é apenas contínua, ver em [Muskhelishvili 53] que ela ainda é válida.

O teorema a seguir é muito importante pois ele nos dá o comportamento das integrais de Cauchy nas vizinhanças dos extremos de $L$.

Teorema 1.3 Seja $L$ um arco aberto com extremos a e b. Sejam $\Phi(z)$ (definida pela fórmula (1.1)) e

$$
\psi(x)=\frac{1}{2 \pi i} f_{L} \frac{\phi(t)}{t-x} d t
$$


onde $\phi$ uma função definida em L, satisfazendo uma das seguintes propriedades:

1. $\phi \in H^{\mu}(L)$.

2. $\phi \in H^{\mu}$ em todo $L$, exceto em um ponto $c \in L$ onde ela tem uma descontinuidade do tipo salto, isto é, $\phi(c-0) \neq \phi(c+0)$.

3. $\phi(x)=\phi^{*}(x)(x-c)^{-\gamma}$, onde $\gamma=\alpha+i \beta, \quad 0 \leq \alpha<1$, sendo $c$ um dos extremos a ou $b$ de $L$ e $\phi^{*} \in H^{\mu}(L)$.

então,

a) Se $\phi$ satisfaz 1), temos

$$
\begin{aligned}
& \Phi(z)=\overline{+} \frac{\phi(c)}{2 \pi i} \ln (z-c)+\Phi_{0}(z) \\
& \psi(x)=\overline{+} \frac{\phi(c)}{2 \pi i} \ln (x-c)+\psi_{0}(x)
\end{aligned}
$$

O sinal superior aparece com $c=a$ e o sinal inferior com $c=b$. As funções $\Phi_{0} e$ $\psi_{0}$ são limitadas na vizinhança de $c$.

b) Se $\phi$ satisfaz 2), temos

$$
\begin{aligned}
& \Phi(z)=\frac{\phi(c-0)-\phi(c+0)}{2 \pi i} \ln (z-c)+\phi_{0}(z) \\
& \psi(x)=\frac{\phi(c-0)-\phi(c+0)}{2 \pi i} \ln (x-c)+\psi_{0}(x)
\end{aligned}
$$

onde $\Phi_{0}$ e $\psi_{0}$ são como em a).

c) Se $\phi$ satisfaz 3) e $\gamma=\alpha+i \beta \neq 0$, temos

$$
\Phi(z)= \pm \frac{e^{ \pm}-\gamma \pi i}{2 i \sin \gamma \pi} \frac{\phi^{*}(c)}{(z-c)^{\gamma}}+\Phi_{0}(z)
$$

e

$$
\psi(x)= \pm \frac{\cot (\gamma \pi)}{2 i} \frac{\phi^{*}(c)}{(x-c)^{\gamma}}+\psi_{0}(x)
$$

onde os sinais, $\Phi_{0}$ e $\psi_{0}$ são como em a).

A prova deste resultado pode ser encontrada em [Muskhelishvili 53]. 


\subsection{O Problema de Hilbert para Arcos Abertos}

Seja $L=L_{1}+L_{2}+\ldots+L_{p}, \operatorname{com} L_{j}$ arcos abertos. Para $j=1,2, \ldots, p$ definimos $c_{2 j-1}=a_{j}$ e $c_{2 j}=b_{j}$ onde $a_{j}$ e $b_{j}$ são os extremos do arco $L_{j}$.

Definição 1.9 Uma função $\Phi(z)$, holomorfa no plano complexo deletado $S$, contínua $\grave{a}$ esquerda e à direita de $L$, com exceção dos extremos $c_{k}$ de $L$, para $k=1,2, \ldots, 2 p e$ satisfazendo na vizinhança destes extremos a condição:

$$
|\Phi(z)| \leq \frac{K}{\left|z-c_{k}\right|^{\alpha}}, 0 \leq \alpha<1 \text { e } K \text { constante }
$$

é chamada função seccionalmente holomorfa com uma linha de descontinuidade $L$.

Definição 1.10 Sejam $G(x)$ e $g(x)$ funções Hölder contínuas em $L, G(x) \neq 0$ em $L$. $O$ problema de Hilbert consiste em encontrar uma função seccionalmente holomorfa $\Phi(z)$, tendo grau finito no infinito e satisfazendo uma das seguintes condições em L :

$$
\begin{gathered}
\Phi^{+}(x)=G(x) \Phi^{-}(x) \\
\Phi^{+}(x)=G(x) \Phi^{-}(x)+g(x) .
\end{gathered}
$$

Os problemas dados por (1.10) e (1.11) são chamados respectivamente Problema Homogêneo $e$ Problema Não Homogêneo.

\subsubsection{Solução do Problema Homogêneo}

Sejam

$$
\Gamma(z)=\frac{1}{2 \pi i} \int_{L} \frac{\ln (G(t))}{t-z} d t
$$

onde $\ln (G(t))$ é qualquer ramo da função multivalente que varia continuamente em cada um dos $\operatorname{arcos} L_{j}$,

$$
\Pi(z)=\left(z-c_{1}\right)^{\lambda_{1}}\left(z-c_{2}\right)^{\lambda_{2}} \ldots\left(z-c_{2 p}\right)^{\lambda_{2 p}}
$$

e $\lambda_{k}$ inteiros satisfazendo a seguinte propriedade:

$$
-1<\alpha_{k}+\lambda_{k}<1
$$

com

$$
\alpha_{k}+i \beta_{k}=\mp \frac{1}{2 \pi i} \ln \left(G\left(c_{k}\right)\right)
$$


Teorema 1.4 Uma função seccionalmente holomorfa $\Phi(z)$ com grau finito no infinito é uma solução do problema homogêneo de Hilbert se, e somente se,

$$
\Phi(z)=P(z) X(z)
$$

onde

$$
X(z)=\Pi(z) e^{\Gamma(z)}
$$

e $P(z)$ é um polinômio.

Na prova deste teorema pode ser visto que $X(z)$ dada por (1.13) só estará completamente determinada com a escolha dos $\lambda_{k}$. Tal escolha está intimamente ligada à classe onde se busca a solução e que por sua vez depende dos conceitos de extremos especiais e não especiais do arco $L$. Escolhida a classe onde se busca a solução, $X(z)$ passa a ser referenciada como solução fundamental desta classe.

Definição 1.11 A função

$$
Z(x)=\Pi(x) e^{\Gamma(x)}, x \in L
$$

onde

$$
\Gamma(x)=\frac{1}{2 \pi i} f_{L} \frac{\ln (G(t))}{t-x} d t
$$

é chamada função fundamental da mesma classe.

Definição 1.12 O inteiro

$$
\kappa=-\sum_{j=1}^{2 p} \lambda_{j}
$$

é chamado índice da classe considerada.

É obvio, de (1.13) e da definição de $\Gamma(z)$, que $X(z)$ tem grau $(-\kappa)$ no infinito e que

$$
\lim _{z \rightarrow \infty} z^{k} X(z)=1
$$

Lema 1.2 Sejam $X(z)$ e $Z(x)$ dadas respectivamente por (1.13) e (1.14). Então

$$
\begin{aligned}
X^{+}(x) & =Z(x) \sqrt{G(x)} \\
X^{-}(x) & =\frac{Z(x)}{\sqrt{G(x)}} \\
Z(x) & =\sqrt{X^{+}(x) X^{-}(x)}
\end{aligned}
$$


onde o ramo de $\sqrt{G(x)}$ é o ramo principal da função logarítmo.

Para a função $Z(x)$ temos também a seguinte expressão:

$$
Z(x)=w_{0}(x)\left(x-c_{1}\right)^{\gamma_{1}}\left(x-c_{2}\right)^{\gamma_{2}} \ldots\left(x-c_{2 p}\right)^{\gamma_{2 p}} \in H^{*},
$$

onde $\gamma_{k}=\alpha_{k}+\lambda_{k}+i \beta_{k}$ e $w_{0}(x)$ satisfaz a condição de Hölder em $L$.

\subsubsection{Solução do Problema não Homogêneo}

Teorema 1.5 Sejam $\kappa$ o indice e $X(z)$ a solução fundamental de (1.10) numa determinada classe.

1- Para $\kappa \geq 0$ a solução de (1.11) da mesma classe, anulando no infinito é dada por:

$$
\Phi(z)=\frac{X(z)}{2 \pi i} \int_{L} \frac{g(t)}{X^{+}(t)(t-z)} d t+X(z) P_{\kappa-1}(z)
$$

onde $P_{\kappa-1}(z)$ é um polinômio de grau $\kappa-1$.

2- Para $\kappa<0$ a solução da dada classe, anulando no infinito existe se, e somente se, as condições

$$
\int_{L} \frac{t^{j} g(t)}{X^{+}(t)} d t=0, \quad j=0,1, \ldots,-\kappa-1
$$

forem satisfeitas e neste caso a solução é dada por (1.15) com $P_{\kappa-1} \equiv 0$.

\subsection{Polinômios Ortogonais de Jacobi}

Definição 1.13 [Szegö 59] Polinômios de Jacobi são polinômios que formam um sistema ortogonal, no intervalo $[-1,1]$ com respeito à função peso $w(x)=(1-x)^{\alpha}(1+x)^{\beta}$, onde $\alpha, \beta>-1$.

Notação: $P_{j}^{(\alpha, \beta)}(x)$ denota o Polinômio de Jacobi de grau $j$ na variável $x$.

São conhecidos pela fórmula de Rodríguez

$$
P_{j}^{(\alpha, \beta)}(x)=\frac{(-1)^{j}}{2^{j} j !}(1-x)^{-\alpha}(1+x)^{-\beta} \frac{d^{j}}{d x^{j}}\left\{(1-x)^{\alpha+j}(1+x)^{\beta+j}\right\} .
$$

Assim

$$
P_{0}^{(\alpha, \beta)}(x)=1 ; P_{1}^{(\alpha, \beta)}(x)=\frac{1}{2}[(\alpha+\beta+2) x+(\alpha-\beta)]
$$


e para $j=2,3,4, \ldots$ satisfazem a seguinte relação de recorrência:

$$
P_{j}^{(\alpha, \beta)}(x)=\left(A_{j} x+B_{j}\right) P_{j-1}^{(\alpha, \beta)}(x)-C_{j} P_{j-2}^{(\alpha, \beta)}(x),
$$

onde:

$$
\begin{aligned}
A_{j} & =\frac{(2 j+\alpha+\beta-1)(2 j+\alpha+\beta)}{2 j(j+\alpha+\beta)}, \\
B_{j} & =\frac{(2 j+\alpha+\beta-1)\left(\alpha^{2}-\beta^{2}\right)}{2 j(j+\alpha+\beta)(2 j+\alpha+\beta-2)} \\
C_{j} & =\frac{(j+\alpha-1)(j+\beta-1)(2 j+\alpha+\beta)}{j(j+\alpha+\beta)(2 j+\alpha+\beta-2)} .
\end{aligned}
$$

Os zeros de $P_{j}^{(\alpha, \beta)}$ estão localizados no interior de intervalo $[-1,1]$ e de acordo com os valores de $\alpha$ e $\beta$, os Polinômios de Jacobi recebem nomes especiais. Por exemplo, quando $\alpha=\beta=-1 / 2$ temos os polinômios de Chebyshev de primeira espécie.

Notação: $T_{n}(x)$ denota o polinômio de Chebyshev de primeira espécie de grau $n$.

No método de colocação polinomial, usaremos como pontos de colocação os zeros de $T_{n+1}(x)$, os quais são dados pela expressão

$$
x_{i}=\cos \frac{(2 i+1) \pi}{2(n+1)}, i=0,1, \ldots, n \text {. }
$$

\subsection{A Função Gama e os números binomiais}

Definição 1.14 [Szegö 59] A função Gama (ou Euleriana de segunda espécie) é definida por

$$
\Gamma(\alpha)=\int_{0}^{\infty} e^{-t} t^{\alpha-1} d t, \Re(\alpha)>0
$$

satisfazendo a seguinte propriedade

$$
\Gamma(\alpha+1)=\alpha \Gamma(\alpha) .
$$

Em [ Abramovitz 64] podemos encontrar a equação que relaciona a função Gama e os números binomiais

$$
\begin{aligned}
\left(\begin{array}{c}
-\alpha \\
k
\end{array}\right) & =\left(\begin{array}{c}
-\alpha \\
-\alpha-k
\end{array}\right) \\
& =(-1)^{k}\left(\begin{array}{c}
k+\alpha-1 \\
k
\end{array}\right)=(-1)^{k} \frac{\Gamma(k+\alpha)}{\Gamma(\alpha) k !}
\end{aligned}
$$




\subsection{Quadratura de Gauss}

Esta quadratura pode ser encontrada em [Monegato 90].

Seja $w(t)=(1-t)^{\alpha}(1+t)^{\beta}$ a função peso definida em $[-1,1], \operatorname{com} \alpha=-\beta=1 / 2$.

A regra de quadratura dada por

$$
\int_{-1}^{1} w(t) f(t) d t \cong \sum_{i=1}^{n} H_{n, i}^{(1 / 2,-1 / 2)} f\left(t_{n, i}\right)
$$

onde $t_{n, i}$ são os zeros do polinômio de Jacobi $P_{n}^{(1 / 2,-1 / 2)}(t)$, dados por

$$
t_{n, i}=\cos \left(\frac{2 i \pi}{2 n+1}\right), i=1, \ldots, n
$$

e

$$
H_{n, i}^{(1 / 2,-1 / 2)}=\frac{2 \pi}{2 n+1}\left(1-t_{n, i}\right)
$$

é chamada quadratura de Gauss com $n$ pontos. 


\section{Capítulo 2}

\section{Equações Integrais Singulares (EIS) com núcleo de Cauchy}

\subsection{Introdução}

O principal objetivo deste capítulo é apresentar a teoria das EIS com núcleo de Cauchy, mostrando com a ajuda da fórmula de Plemelj-Sokhotsky, a equivalência entre o Problema de Hilbert e a resolução de EIS com coeficientes constantes. Os detalhes destes assuntos podem ser vistos em [Cuminato 87].

\subsection{Equação Integral Singular}

Seja $L$ uma união de arcos abertos $L_{j}, \mathrm{j}=1,2, \ldots, \mathrm{p}$ com extremos $a_{j}$ e $b_{j}$ e direção positiva indo de $a_{j}$ para $b_{j}$.

Seja a equação integral

$$
a(x) \phi(x)+\frac{b(x)}{\pi} f_{L} \frac{\phi(t)}{t-x} d t+\int_{L} m(x, t) \phi(t) d t=f(x), \quad x \in L
$$

onde $a, b, m$ e $f$ são funções reais e satisfazem a condição de Hölder em $L$.

Vamos mostrar a equivalência de uma tal equação com um problema de Hilbert não homogêneo, tendo assim, sua solução dada pelo teorema 1.5.

Para isto sejam os operadores

$$
A \phi(x)=a(x) \phi(x)+\frac{b(x)}{\pi} f_{L} \frac{\phi(t)}{t-x} d t
$$


e

$$
M \phi(x)=\int_{L} m(x, t) \phi(t) d t
$$

As equações

$$
A \phi=f
$$

e

$$
A \phi+M \phi=f
$$

são chamadas respectivamente equação dominante e equação completa.

\subsubsection{Solução da Equação Dominante}

Consideremos a equação dominante (2.2). Seja $\Phi(z)$ dada por (1.1).

Substituindo (1.7) e (1.8) em (2.2), temos

$$
a(x)\left[\Phi^{+}(x)-\Phi^{-}(x)\right]+i b(x)\left[\Phi^{+}(x)+\Phi^{-}(x)\right]=f(x)
$$

ou

$$
\Phi^{+}(x)=\frac{a(x)-i b(x)}{a(x)+i b(x)} \Phi^{-}(x)+\frac{f(x)}{a(x)+i b(x)}
$$

De (1.1) temos que $\Phi(z)$ tem grau (-1) no infinito e assumindo que

$$
a(x)-i b(x) \neq 0 \quad \text { e } \quad a(x)+i b(x) \neq 0
$$

ou equivalentemente

$$
r(x)=\sqrt{a^{2}(x)+b^{2}(x)} \neq 0
$$

então a função $G(x) \neq 0$ para todo $x \in L$ e é Hölder contínua.

Assim (2.4) é um problema não homogêneo de Hilbert com

$$
G(x)=\frac{a(x)-i b(x)}{a(x)+i b(x)}
$$

Podemos então usar o teorema 1.5 e a solução de (2.4) será dada por :

$$
\Phi(z)=\frac{X(z)}{2 \pi i} \int_{L} \frac{f(t)}{(a(t)+i b(t)) X^{+}(t)(t-z)} d t+X(z) P_{\kappa-1}(z) .
$$

Usando o lema 1.2 , temos 


$$
r(x) Z(x)=\frac{r(x) X^{+}(x)}{\sqrt{G(x)}}=(a(x)+i b(x)) X^{+}(x)
$$

Substituindo este resultado em (2.5), temos

$$
\Phi(z)=\frac{X(z)}{2 \pi i} \int_{L} \frac{f(t)}{r(t) Z(t)(t-z)} d t+X(z) P_{\kappa-1}(z) .
$$

Podemos escrever a equação acima da seguinte maneira:

$$
\Phi(z)=X(z) \Psi(z)+X(z) P_{\kappa-1}(z)=X(z)\left[\Psi(z)+P_{k-1}(z)\right]
$$

com

$$
\Psi(z)=\frac{1}{2 \pi i} \int_{L} \frac{f(t)}{r(t) Z(t)(t-z)} d t .
$$

Pelo uso das fórmulas de Plemelj-Sokhotsky (1.4) e (1.5) para $\Psi(z)$ temos:

$$
\begin{aligned}
& \Psi^{+}(x)=\frac{1}{2} \frac{f(x)}{r(x) Z(x)}+\frac{1}{2 \pi i} f_{L} \frac{f(t)}{r(t)) Z(t)(t-x)} d t \\
& \Psi^{-}(x)=\frac{-1}{2} \frac{f(x)}{r(x) Z(x)}+\frac{1}{2 \pi i} f_{L} \frac{f(t)}{r(t) Z(t)(t-x)} d t
\end{aligned}
$$

Estamos agora em condições de dar uma expressão para $\phi(x)$ (solução da equação (2.2)). Considerando que $\Phi(z)$ é dada por (2.6) e satisfaz (1.8), obtemos:

$$
\phi(x)=X^{+}(x)\left[\Psi^{+}(x)+P_{k-1}(x)\right]-X^{-}(x)\left[\Psi^{-}(x)+P_{k-1}(x)\right] .
$$

Substituindo (2.7) e (2.8) em (2.9), vem

$$
\begin{aligned}
\phi(x)= & \frac{1}{2} \frac{f(x)}{r(x) Z(x)}\left[X^{+}(x)+X^{-}(x)\right]+\frac{1}{2 \pi i} f_{L} \frac{f(t)}{r(t) Z(t)(t-x)} d t\left[X^{+}(x)-X^{-}(x)\right] \\
& +P_{k-1}(x)\left[X^{+}(x)-X^{-}(x)\right]
\end{aligned}
$$

Do lema 1.2 , podemos ver que

$$
\begin{aligned}
X^{+}(x)+X^{-}(x) & =\sqrt{G(x)} Z(x)+\frac{Z(x)}{\sqrt{G(x)}} \\
& =\left[\sqrt{\frac{a(x)-i b(x)}{a(x)+i b(x)}}+\sqrt{\frac{a(x)+i b(x)}{a(x)-i b(x)}}\right] Z(x) \\
& =\frac{2 a(x) Z(x)}{r(x)}
\end{aligned}
$$




$$
X^{+}(x)-X^{-}(x)=-\frac{2 i b(x) Z(x)}{r(x)}
$$

resultando, quando $\kappa \geq 0$, a seguinte expressão para a solução da equação dada

$$
\phi(x)=\frac{a(x) f(x)}{r^{2}(x)}-\frac{b(x) Z(x)}{r(x) \pi} f_{L} \frac{f(t)}{r(t) Z(t)(t-x)} d t-\frac{2 i b(x) Z(x)}{r(x)} P_{k-1}(x) .
$$

Se $\kappa<0$, a solução dada por (2.10) com $P_{k-1} \equiv 0$ existe se, e somente se, as condições:

$$
\int_{L} \frac{t^{j} f(t)}{r(t) Z(t)} d t=0, \quad j=0,1, \ldots,-\kappa-1,
$$

forem satisfeitas. Note que estas condições seguem de (1.16).

Demonstramos assim o seguinte

Teorema 2.1 A solução $\phi$ da equação dominante (2.2) é dada por:

$$
\begin{array}{lr}
\phi(x)=A^{I} f(x)-\frac{2 i b(x) Z(x)}{r(x)} P_{\kappa-1}(x) & \text { se } \kappa \geq 0 \\
\phi(x)=A^{I} f(x) & \text { se } \kappa<0,
\end{array}
$$

onde

$$
A^{I} f(x)=\frac{a(x) f(x)}{r^{2}(x)}-\frac{b(x) Z(x)}{r(x) \pi} f_{L} \frac{f(t)}{r(t) Z(t)(t-x)} d t
$$

e para $\kappa<0$, a solução existe se, e somente se, forem válidas as condições

$$
\int_{L} \frac{t^{j} f(t)}{r(t) Z(t)} d t=0, \quad j=0,1, \ldots,-\kappa-1
$$

Corolário 2.1 Para toda função $f$ que satisfaça a condição de Hölder em L temos:

$$
\begin{array}{ll}
A A^{I} f=f+\frac{b(x) Z(x)}{r(x)} P_{\kappa-1}(x), & \text { se } \kappa \geq 0 \\
A A^{I} f=f & \text { se } \kappa<0 .
\end{array}
$$

Para $\kappa \geq 0$, o núcleo do operador A tem dimensão $\kappa$ e quando $\kappa \geq 1$ é gerado pelo seguinte conjunto de funções:

$$
\frac{b(x) Z(x)}{r(x)}\left\{1, x, x^{2}, \ldots, x^{\kappa-1}\right\}
$$




\subsubsection{Solução da Equação Completa}

Teorema 2.2 A solução da equação completa (2.3) é também solução da equação:

$$
\phi(x)+\int_{L} N(x, t) \phi(t) d t=f_{1}(x)
$$

onde

$$
\begin{aligned}
N(x, t) & =\frac{a(x) m(x, t)}{r^{2}(x)}-\frac{b(x) Z(x)}{r(x) \pi} f_{L} \frac{m(s, t)}{r(s) Z(s)(s-x)} d s \\
f_{1}(x) & =\frac{a(x) f(x)}{r^{2}(x)}-\frac{b(x) Z(x)}{r(x) \pi} f_{L} \frac{f(t)}{r(t) Z(t)(t-x)} d t-\frac{b(x) Z(x)}{r(x)} P_{k-1}(x)
\end{aligned}
$$

Prova: Usando a notação de operadores para (2.3)

$$
A \phi+M \phi=f
$$

ou

$$
A \phi=f-M \phi
$$

podemos afirmar que $f-M \phi$ é Hölder contínua, uma vez que $f(x)$ e $m(x, t)$ satisfazem a condição de Hölder em $L$ e $\phi$ é integrável por exigência do problema de Hilbert. Assumindo que o lado direito de (2.12) é conhecido e usando o teorema 2.1, o resultado segue.

$\mathrm{Na}$ forma de operadores temos:

$$
\phi(x)+A^{I} M \phi(x)=A^{I} f(x)+\frac{b(x) Z(x)}{r(x)} P_{k-1}(x)
$$

onde

$$
\begin{gathered}
A^{I} M \phi(x)=\int_{L} N(x, t) \phi(t) d t \\
f_{1}(x)=A^{I} f(x)+\frac{b(x) Z(x)}{r(x)} P_{k-1}(x)
\end{gathered}
$$

Em [Cuminato 87] está demonstrado que a equação (2.11) pode ser transformada numa equação integral de Fredholm, o que garante a existência de uma única solução, desde que -1 não seja um autovalor do núcleo da equação transformada.

O teorema a seguir é importante, pois apoia-se nele toda a teoria do método de colocação polinomial como veremos no capítulo seguinte. 
Teorema 2.3 Seja $\phi(x)$ a solução da equação dominante (2.2)e $X(z)$ a solução fundamental do Problema de Hilbert associado, ilimitada nos extremos de L. Entẫo, para $-1<x<1, \phi(x)$ pode ser escrita da seguinte forma:

$$
\phi(x)=w(x) g(x)
$$

com $w(x)=Z(x) / r(x)$ e $g(x)$ Hölder contínua. A mesma expressão continua válida se $\phi(x)$ é solução da equação completa.

Prova: Consideremos, sem perda de generalidade, $L=[-1,1]$. Do teorema 2.1, temos

$$
\phi(x)=\frac{a(x) f(x)}{r^{2}(x)}-\frac{Z(x)}{r(x)}\left[\frac{1}{\pi} f_{-1}^{1} \frac{f(t) b(x)}{r(t) Z(t)(t-x)} d t+b(x) P_{\kappa-1}(x)\right]
$$

Adicionando e subtraindo $f(x) b(t)$ no integrando da expressão acima, temos

$$
\phi(x)=\frac{a(x) f(x)}{r^{2}(x)}-\frac{Z(x)}{r(x)}\left[\frac{1}{\pi} f_{-1}^{1} \frac{f(t) b(x)+f(x) b(t)-f(x) b(t)}{r(t) Z(t)(t-x)} d t+b(x) P_{k-1}(x)\right]
$$

ou

$$
\begin{aligned}
\phi(x) & =\frac{a(x) f(x)}{r^{2}(x)}-\frac{Z(x)}{r(x)}\left[\frac{f(x)}{\pi} f_{-1}^{1} \frac{b(t)}{r(t) Z(t)(t-x)} d t\right. \\
& \left.+\frac{1}{\pi} f_{-1}^{1} \frac{f(t) b(x)-f(x) b(t)}{r(t) Z(t)(t-x)} d t+b(x) P_{k-1}(x)\right]
\end{aligned}
$$

Mas,

$$
\frac{1}{\pi} f_{-1}^{1} \frac{b(t)}{r(t) Z(t)(t-x)} d t=\frac{a(x)}{r(x) Z(x)}-p p\left(X^{-1}, x\right)
$$

onde $p p\left(X^{-1}, x\right)$ é um polinômio de grau $\kappa$ para $\kappa \geq 0$ e $p p\left(X^{-1}, x\right)=0$ para $\kappa<0$.

Substituindo (2.15) em (2.14), obtemos

$$
\begin{aligned}
\phi(x)= & \frac{a(x) f(x)}{r^{2}(x)}-\frac{Z(x)}{r(x)}\left[f(x)\left(\frac{a(x)}{r(x) Z(x)}-p p\left(X^{-1}, x\right)\right)\right. \\
& \left.+\frac{1}{\pi} f_{-1}^{1} \frac{f(t) b(x)-f(x) b(t)}{r(t) Z(t)(t-x)} d t+b(x) P_{k-1}(x)\right] \\
& =\frac{Z(x)}{r(x)}\left[f(x) p p\left(X^{-1}, x\right)+\frac{1}{\pi} f_{-1}^{1} \frac{f(x) b(t)-f(t) b(x)}{r(t) Z(t)(t-x)} d t+b(x) P_{k-1}(x)\right]
\end{aligned}
$$


Substituindo na expressão acima a identidade

$$
f(x) b(t)-f(t) b(x)=f(x)[b(t)-b(x)]-b(x)[f(t)-f(x)]
$$

resulta:

$$
\begin{aligned}
\phi(x)= & \frac{Z(x)}{r(x)}\left\{f(x)\left[p p\left(X^{-1}, x\right)+\frac{1}{\pi} \int_{-1}^{1} \frac{b(t)-b(x)}{r(t) Z(t)(t-x)} d t\right]\right. \\
& \left.+b(x)\left[\frac{-1}{\pi} \int_{-1}^{1} \frac{f(t)-f(x)}{r(t) Z(t)(t-x)} d t+P_{k-1}(x)\right]\right\} .
\end{aligned}
$$

Examinando (2.16), concluímos que

$$
\phi(x)=w(x) g(x), \text { onde } w(x)=\frac{Z(x)}{r(x)} \text { e } g(x) \text { Hölder contínua. }
$$

De fato, como optamos pela pesquisa de solução ilimitada em ambos os extremos, a função $1 / Z(x)$ é limitada para todo $x \in[-1,1]$ e anula-se em tais extremos. Além disto, as funções $f(x)$ e $b(x)$ sendo Hölder contínuas,

$$
\frac{1}{\pi} \int_{-1}^{1} \frac{b(t)-b(x)}{r(t) Z(t)(t-x)} d t
$$

$\mathrm{e}$

$$
\frac{1}{\pi} \int_{-1}^{1} \frac{f(t)-f(x)}{r(t) Z(t)(t-x)} d t
$$

satisfazem a condição de Hölder em L, ficando demonstrada a primeira parte do teorema.

Se estivermos procurando solução limitada nos extremos, vemos que a mesma pode ainda ser decomposta na forma dada por (2.13). A diferença é que agora não podemos garantir que $g(x)$ seja Hölder contínua.

Agora, se $\phi(x)$ é solução da equação completa, provamos a validade da expressão (2.13), usando exatamente a mesma técnica empregada na determinação da solução. Isto equivale a tomar o termo que envolve o operador compacto do lado direito e tratá-lo como conhecido. Isto feito, a segunda parte do teorema segue com o uso do fato de que a função $M \phi(x)$, e conseqüentemente todo o lado direito da equação completa, satisfaz a condição de Hölder em L. 


\section{Capítulo 3}

\section{Equação integral singular com coeficientes constantes}

\subsection{Introdução}

Neste capítulo será feita uma exposição dos resultados básicos da teoria das EIS com coeficientes constantes, usando os resultados do capítulo anterior. A seguir, apresentamos o método de colocação polinomial, e deduzimos a sua equação básica. A interpolação polinomial e o operador projeção fornecem resultados importantes que auxiliam no estabelecimento da convergência do método.Finalmente, enunciamos o teorema de convergência. Estes assuntos podem ser encontrados em [Cuminato 87].

\subsection{A equação integral}

Considere a equação integral singular

$$
a \phi(x)+\frac{b}{\pi} f_{-1}^{1} \frac{\phi(t) d t}{t-x}+\int_{-1}^{1} k(x, t) \phi(t) d t=f(x),-1<x<1
$$

onde $k$ e $f$ são funções Hölder contínuas, os coeficientes $a$ e $b$ da equação são constantes reais e $\phi$ é a solução procurada.

É conveniente considerar, sem perda de generalidade, a normalização $a^{2}+b^{2}=1$, isto é, $r=1$.

A primeira integral da equação (3.1) é entendida no sentido do valor principal 
de Cauchy. Devido a isto, os pontos que podem causar singularidades na solução são os extremos -1 e 1. É importante ressaltar que isto pode acontecer mesmo que o integrando seja regular (ver teorema 1.3).

A equação ( 3.1 ) pode, em geral, não possuir solução ou possuir infinitas soluções, dependendo da classe de funções na qual ela for resolvida.

Desta forma, a solução de (3.1) só faz sentido se fixarmos o conjunto no qual se busca uma solução. De acordo com Muskhelishvili são três as classes de solução possíveis:

a) Funções limitadas em $[-1,1]$.

b) Funções limitadas em um dos extremos -1 ou 1 .

c) Funções ilimitadas em ambos os extremos -1 e 1.-

Resultados apresentados anteriormente garantem que, para a equação $(3.1), G(x)$ é uma função constante dada por

$$
G(x)=\frac{a-i b}{a+i b}
$$

Assim,

$$
\begin{gathered}
\Gamma(x)=\frac{1}{2 \pi i} \ln \left(\frac{a-i b}{a+i b}\right) f_{-1}^{1} \frac{d t}{t-x}=\frac{1}{2 \pi i} \ln \left(\frac{a-i b}{a+i b}\right) \ln \left(\frac{1-x}{1+x}\right) \\
e^{\Gamma(x)}=(1-x)^{\gamma}(1+x)^{-\gamma} \text { onde } \gamma=\frac{1}{2 \pi i} \ln \left(\frac{a-i b}{a+i b}\right)
\end{gathered}
$$

e

$$
\Pi(x)=(1-x)^{M}(1+x)^{N},
$$

onde $M$ e $N$ são inteiros escolhidos de modo que a solução esteja em uma das classes acima e seja integrável.

Resulta então, para $Z(x)$, a seguinte expressão

$$
Z(x)=(1-x)^{M}(1+x)^{N}(1-x)^{\gamma}(1+x)^{-\gamma}=(1-x)^{\alpha}(1+x)^{\beta}
$$

com

$$
\begin{gathered}
\alpha=\gamma+M \\
\beta=-\gamma+N
\end{gathered}
$$

A solução $\phi(x)$ pode ser decomposta como em (2.13) com $g(x)$ Hölder contínua e $w(x)=Z(x)$ 
indice

Dada a equação integral (3.1) e fixada a classe de soluções podemos definir o

$$
\kappa=-(\alpha+\beta)=-(M+N)
$$

relativo à classe dada.

O objetivo de assegurar singularidades integráveis, garantido pelas condições:

$$
\begin{aligned}
& -1<\gamma+M<1 \\
& -1<-\gamma+N<1
\end{aligned}
$$

faz com que o índice fique restrito a $-1,0,1$.

No caso em que

(a) $\kappa=1$, a solução é ilimitada em ambos os extremos -1 e 1. Neste caso a equação tem infinitas soluçôes e a unicidade de solução da equação integral só pode ser obtida com o uso de uma condição suplementar, que usualmente é da forma:

$$
\int_{-1}^{1} \phi(x) d x=C
$$

onde $C$ é uma constante.

(b) $\kappa=0$, a equação integral possui uma única solução.

(c) $\kappa=-1$, a solução é limitada em ambos os extremos e, se existir, deve satisfazer a condição de consistência:

$$
\int_{-1}^{1} \frac{1}{w(x)}\left[a \phi(x)+\frac{b}{\pi} f_{-1}^{1} \frac{\phi(t)}{t-x} d t\right] d x=0 .
$$

Assumindo, de agora em diante, que o índice e a classe de solução são dados e que $\phi(x)$ tem a forma dada pela equação (2.13), podemos pensar na solução numérica da equação integral (3.1). Das considerações acima (3.1)pode ser reescrita da seguinte maneira

$$
a w(x) g(x)+\frac{b}{\pi} f_{-1}^{1} \frac{w(t) g(t)}{t-x} d t+\int_{-1}^{1} k(x, t) w(t) g(t) d t=f(x),-1<x<1 .
$$

$\mathrm{Na}$ notação de operadores temos

$$
H g+K g=f,
$$

onde

$$
H g=a w(x) g(x)+\frac{b}{\pi} f_{-1}^{1} \frac{w(t) g(t)}{t-x} d t
$$


é chamado Operador Singular e

$$
K g=\int_{-1}^{1} k(x, t) w(t) g(t) d t
$$

é chamado Operador Regular.

Define-se o operador $H^{I}$ por

$$
H^{I} f=a w^{*}(x) f(x)-\frac{b}{\pi} f_{-1}^{1} \frac{w^{*}(t) f(t)}{t-x} d t,
$$

onde $w^{*}(x)=[w(x)]^{-1}$.

Mas

$$
H^{I} H g= \begin{cases}g-g_{0} & \text { se } \kappa=1 \\ g & \text { se } \kappa \leq 0\end{cases}
$$

para toda função Hölder contínua $g$ com $g_{0}$ constante e pertencente ao núcleo de $H$.

Aplicando $H^{I}$ à esquerda de (3.5), temos

$$
\begin{aligned}
& g+H^{I} K g=H^{I} f+g_{0} \text { se } \kappa=1 \\
& g+H^{I} K g=H^{I} f \quad \text { se } \kappa \leq 0
\end{aligned}
$$

Se $k(x, t)$ é Hölder contínua então $H^{I} K$ é um operador de Fredholm e as equações (3.9) são equações de Fredholm de segunda espécie, tendo uma única solução para cada $g_{0}$ fixo, se -1 não for um autovalor de $H^{I} K$, condição esta que será assumida de agora em diante.

As equações (3.9) podem ser escritas na forma compacta

$$
g+H^{I} K g=H^{I} f+g_{0} \delta_{1, k}
$$

onde $\delta_{i, j}$ é o delta de Kronecker.

Usando em (3.10) o fato de que $\left(I+H^{I} K\right)$ é inversível, $g$ pode ser dada pela seguinte expressão :

$$
g=\left(I+H^{I} K\right)^{-1}\left(H^{I} f+g_{0} \delta_{1, k}\right)
$$

No caso em que $\kappa=1$, podemos determinar $g_{0}$ multiplicando a equação acima por $w(x)$, integrando sobre $[-1,1]$ e usando a condição suplementar da forma dada por (3.2). Assim temos

$$
\int_{-1}^{1} w(x) g(x) d x=\int_{-1}^{1} \phi(x) d x=\text { constante }
$$


ou

$$
\int_{-1}^{1} w(x)\left(I+H^{I} K\right)^{-1} H^{I} f(x) d x+g_{0} \int_{-1}^{1}\left(I+H^{I} K\right)^{-1} 1(x) d x=\text { constante. }
$$

Para que $g_{0}$ possa ser unicamente determinado devemos ter

$$
\int_{-1}^{1}\left(I+H^{I} K\right)^{-1} 1(x) d x \neq 0
$$

condição esta que podemos assumir sempre válida, onde $1(x)$ significa a função constante $f(x)=1$.

O lema que vem a seguir tem sua aplicação no método de colocação polinomial.

Lema 3.1 [Krenk 75] Sejam $\phi_{i}(x)=P_{i}^{(\alpha, \beta)}(x)$ e $\phi_{i}^{*}(x)=P_{i}^{(-\alpha,-\beta)}(x)$, tal que $\left\{\phi_{i}\right\}$ e $\left\{\phi_{i}^{*}\right\}$ formam sequências de polinômios ortogonais com respeito aos pesos $w(x)$ e $w^{*}(x)$.

Seja ainda $\kappa$ o índice da equação (3.1).

Então,

$$
\begin{gathered}
a w(x) \phi_{i}(x)+\frac{b}{\pi} f_{-1}^{1} \frac{w(t) \phi_{i}(t)}{t-x} d t=\frac{-b 2^{-\kappa}}{\sin \pi \alpha} \phi_{i-\kappa}^{*}(x), \\
a w^{*}(x) \phi_{i}^{*}(x)-\frac{b}{\pi} f_{-1}^{1} \frac{w^{*}(t) \phi_{i}^{*}(t)}{t-x} d t=\frac{-b 2^{-\kappa}}{\sin \pi \alpha} \phi_{i+\kappa}(x),
\end{gathered}
$$

onde $\phi_{i} \equiv \phi_{i}^{*} \equiv 0$, para $i<0$.

\subsection{Método de Colocação Polinomial}

Retomemos a equação ( 3.4$)$ e consideremos a aproximação $g_{n}(x)$ de $g(x)$, onde

$$
g_{n}(x)=\sum_{j=0}^{n} a_{j} \phi_{j}(x)
$$

$\phi_{j}(x)=P_{j}^{(\alpha, \beta)}(x)$ são polinômios de Jacobi de grau j e $a_{j}, j=0,1,2, \ldots, n$ são constantes desconhecidas.

Se $g_{n}(x)$ for substituída diretamente na equação integral acima, não é certo que se consiga encontrar os coeficientes $a_{0}, a_{1}, \ldots, a_{n}$ de modo a satisfazer a igualdade. Se isto fosse possível, teríamos a solução exata. 
Para determinar estes coeficientes formamos o resíduo

$$
\begin{aligned}
r_{n}(x) & =\left(H g_{n}+K g_{n}-f\right)(x) \\
& =a w(x) g_{n}(x)+\frac{b}{\pi} f_{-1}^{1} \frac{w(t) g_{n}(t)}{t-x} d t+\int_{-1}^{1} w(t) k(x, t) g_{n}(t) d t-f(x) \\
& =\sum_{j=0}^{n} a_{j}\left[a w(x) \phi_{j}(x)+\frac{b}{\pi} f_{-1}^{1} \frac{w(t) \phi_{j}(t)}{t-x} d t+\int_{-1}^{1} w(t) k(x, t) \phi_{j}(t) d t\right]-f(x) .
\end{aligned}
$$

Usando a propriedade dos polinômios ortogonais dada pela equação (3.12), temos

$$
r_{n}(x)=\sum_{j=0}^{n} a_{j}\left[\frac{-b 2^{-\kappa}}{\sin \pi \alpha} \phi_{j-\kappa}^{*}(x)+\int_{-1}^{1} w(t) k(x, t) \phi_{j}(t) d t\right]-f(x) .
$$

Esta equação, é a equação básica do método de colocação polinomial.

$\mathrm{O}$ método de colocação polinomial consiste fundamentalmente na escolha de pontos distintos $x_{0}, x_{1}, \ldots, x_{n-\kappa} \in[-1,1]$ e na imposição das condições

$$
r_{n}\left(x_{m}\right)=0 ; \quad m=0,1, \ldots, n-\kappa .
$$

obtendo assim um sistema de equações lineares.

Quando

(a) $\kappa=1$, este sistema tem $n$ equações e $n+1$ incógnitas $a_{0}, a_{1}, \ldots, a_{n}$. Para resolvê-lo usamos uma equação adicional, ou seja, exigimos que $g_{n}(x)$ satisfaça a condição suplementar dada pela equação (3.2).

(b) $\kappa=0$, temos $n+1$ equações em $n+1$ incógnitas e o sistema pode ser resolvido.

(c) $\kappa=-1$, temos $n+2$ equações em $n+1$ incógnitas e uma das equações deve ser abandonada para que o sistema possa ser resolvido. A descrição de como isto deve ser feito pode ser encontrada em [Cuminato 90].

Apresentaremos a seguir um lema que apesar da sua simplicidade, e até mesmo da simplicidade da sua prova, é de extrema importância no tratamento da convergência do método de colocação polinomial. Para que este lema apareça de forma natural e simples, definimos na próxima seção, interpolação polinomial e o operador projeção. 


\subsubsection{Interpolação polinomial e operador projeção}

Dados $n-\kappa+1$ pontos distintos $x_{0}, x_{1}, \ldots, x_{n-\kappa}$, pertencentes a $[-1,1]$, o polinômio interpolador $I_{n-\kappa}$ de uma função $f \in C[-1,1]$ sobre os pontos $x_{0}, x_{1}, \ldots, x_{n-\kappa}$ é o único polinômio de grau $n-\kappa$ tal que:

$$
I_{n-\kappa}\left(x_{m}\right)=f\left(x_{m}\right) \quad m=0,1, \ldots, n-\kappa .
$$

O polinômio interpolador pode ser representado na forma de Lagrange:

$$
I_{n-\kappa}(x)=\sum_{m=0}^{n-\kappa} L_{m}(x) f\left(x_{m}\right)
$$

onde

$$
L_{m}(x)=\frac{\left(x-x_{0}\right)\left(x-x_{1}\right) \ldots\left(x-x_{m-1}\right)\left(x-x_{m+1}\right) \ldots\left(x-x_{n-\kappa}\right)}{\left(x_{m}-x_{0}\right)\left(x_{m}-x_{1}\right) \ldots\left(x_{m}-x_{m-1}\right)\left(x_{m}-x_{m+1}\right) \ldots\left(x_{m}-x_{n-\kappa}\right)}
$$

e satisfaz a propriedade $L_{m}\left(x_{k}\right)=\delta_{m k}$ para $k=0,1, \ldots, n-\kappa$, sendo $\delta_{m k}$ o delta de Kronecker.

Definição 3.1 Dado o conjunto de pontos $x_{0}, x_{1}, \ldots, x_{n-\kappa}$, definimos o operador prajeção $P_{n-\kappa}$ por:

$$
\begin{aligned}
P_{n-\kappa}: C[-1,1] & \longrightarrow C[-1,1] \\
f & \longrightarrow P_{n-\kappa} f=\sum_{m=0}^{n-\kappa} L_{m}(x) f\left(x_{m}\right)
\end{aligned}
$$

Lema $3.2 r_{n}\left(x_{m}\right)=0 ; \quad m=0,1, \ldots, n-\kappa$ se e somente se $P_{n-\kappa} r_{n} \equiv 0$.

Prova Seja $r_{n}\left(x_{m}\right)=0, \quad m=0,1, \ldots, n-\kappa$. Da definição de $P_{n-\kappa}$ temos:

$$
P_{n-\kappa} r_{n}(x)=\sum_{m=0}^{n-\kappa} L_{m}(x) r_{n}\left(x_{m}\right) \equiv 0 .
$$

Por outro lado, de $P_{n-\kappa} r_{n} \equiv 0$ vem que $P_{n-\kappa} r_{n}\left(x_{k}\right)=0$ para qualquer k.

Mas

$$
P_{n-\kappa} r_{n}\left(x_{k}\right)=\sum_{m=0}^{n-\kappa} L_{m}\left(x_{k}\right) r_{n}\left(x_{m}\right)=\sum_{m=0}^{n-\kappa} \delta_{m k} r_{n}\left(x_{m}\right)=r_{n}\left(x_{k}\right)
$$


Então

$$
r_{n}\left(x_{k}\right)=0, k=0, \ldots, n-\kappa .
$$

Acabamos de concluir que o conjunto de equações lineares formado por $r_{n}\left(x_{m}\right)=$ $0, m=0,1, \ldots, n-\kappa$, é exatamente o mesmo de $P_{n-\kappa} r_{n} \equiv 0$.

Usando este lema, podemos reescrever o sistema de equações

$$
r_{n}\left(x_{m}\right)=0
$$

como

$$
0=P_{n-\kappa} r_{n}=P_{n-\kappa}\left(H g_{n}+K g_{n}-f\right)
$$

ou seja,

$$
P_{n-\kappa} H g_{n}+P_{n-\kappa} K g_{n}=P_{n-\kappa} f
$$

Das propriedades dos polinômios ortogonais dadas pelo lema (3.1), temos que $H g_{n}$ é um polinômio de grau no máximo $n-\kappa$. Então

$$
P_{n-\kappa} H g_{n}=H g_{n}
$$

e a equação (3.16) toma a forma

$$
H g_{n}+P_{n-\kappa} K g_{n}=P_{n-\kappa} f
$$

Lema 3.3 Seja $k(x, t)$ uma função Hölder contínua e para $t \in[-1,1]$ seja $k_{n}(x, t)$ o polinômio que interpola a funçấo $k(x, t)$ na variável $x$, sobre o conjunto de pontos $y_{0}, y_{1}, \ldots, y_{n}$.

Sejam também os operadores $K$ e $K_{n}$, definidos por

$$
\begin{gathered}
K f(x)=\int_{-1}^{1} k(x, t) f(t) d t \\
K_{n} f(x)=\int_{-1}^{1} k_{n}(x, t) f(t) d t
\end{gathered}
$$

então

$$
P_{n} K=K_{n}
$$

A prova pode ser encontrada em [Cuminato 87]. 


\subsubsection{Teorema de Convergência}

Teorema 3.1 Seja $f \in C^{p}[-1,1] \operatorname{com} f^{(p)} \in H^{\mu}[-1,1], p \geq 0$. Seja $k(x, t)$ uma função Hölder contínua, tal que $k(x, t) \in C^{q}[-1,1]$ com respeito à variável $x$ e $\partial^{q} k(x, t) / \partial x^{q}$ $\in H^{\sigma}[-1,1]$, uniformemente na variável $t, q \geq 0$, isto é,

$$
\left|\frac{\partial^{q} k\left(x_{1}, t\right)}{\partial x^{q}}-\frac{\partial^{q} k\left(x_{2}, t\right)}{\partial x^{q}}\right| \leq M\left|x_{1}-x_{2}\right|^{\sigma}
$$

onde as constantes $M$ e $\sigma$ são independentes de $t$.

Seja também $g(x)$ a solução da seguinte equação integral singular

$$
H g+K g=f .
$$

então para $n$ suficientemente grande a equação

$$
H g_{n}+P_{n} K g_{n}=P_{n} f
$$

tem uma solução $e$,

$$
\max _{x \in[-1,1]}\left|g(x)-g_{n}(x)\right| \leq C n^{-r}
$$

onde $r=\min \{p+\mu, q+\sigma\}-\xi, \xi>0$ arbitrário e $C$ é constante.

A prova do teorema no caso em que o índice $\kappa=0$ ou $\kappa=1$ pode ser vista em [Cuminato 87]. No caso em que o índice $\kappa=-1$, vale um resultado semelhante e pode ser visto em [Cuminato 90]. 


\section{Capítulo 4}

\section{Fórmulas de Quadratura}

\subsection{Introdução}

Este capítulo trata da aproximação numérica das integrais de Cauchy no intervalo $[-1,1]$ tendo por base os trabalhos de Gerasoulis e Cuminato (ver [Gerasoulis 86] e [Cuminato 87] ).

Apresentamos a quadratura de Gerasoulis e analisamos o erro de truncamento da série gerada pelo método, a fim de que dentro de uma dada tolerância de erro $\epsilon$, possamos trabalhar com o menor número de termos possível.

Em aplicações numéricas mostramos o número de termos necessários para resolver uma integral com uma determinada precisão.

Em algorítmo do Ponto Médio, vemos como este número de termos pode ser reduzido sem prejuízo do erro de arredondamento.

Finalmente, mostramos uma regra de quadratura para $S_{i}(x)$.

\subsection{A Fórmula de Gerasoulis}

Definição 4.1 Seja

$$
w^{*}(x)=(1-x)^{-\alpha}(1+x)^{-\beta}
$$

onde $\alpha, \beta \in(-1,1)$. 
Definimos $I_{i}$ e $L_{i}(x)$ pelas integrais:

$$
\begin{gathered}
I_{i}=\int_{x_{i}}^{x_{i+1}} w^{*}(t) d t \\
L_{i}(x)=\int_{x_{i}}^{x_{i+1}} \frac{w^{*}(t)}{t-x} d t \quad x \notin\left[x_{i}, x_{i+1}\right]
\end{gathered}
$$

onde $x_{i}, x_{i+1} \in(-1,1), x_{i}<x_{i+1}$.

Em todo este capítulo, $\Gamma$ é a função definida por (1.19).

\subsubsection{Cálculo de $I_{i}$}

Teorema 4.1 A integral $I_{i}$, definida em (4.1), pode ser avaliada de (a), (b) e (c) abaixo:

(a) Se $0 \leq x_{i}<x_{i+1}$ e $p$ é um ponto arbitrário de $[0,1]$

$$
\begin{gathered}
I_{i}=(1+p)^{-\beta} \sum_{k=0}^{\infty}(-1)^{k} \frac{\Gamma(k+\beta)}{\Gamma(\beta) k !(1+p)^{k}} I_{i, k} \\
I_{i, k}=C\left\{k(1-p) I_{i, k-1}-\left[\left(1-x_{i+1}\right)^{1-\alpha}\left(x_{i+1}-p\right)^{k}-\left(1-x_{i}\right)^{1-\alpha}\left(x_{i}-p\right)^{k}\right]\right\} \\
\text { para } k=0,1,2, \ldots \text { onde } C=1 /(k-\alpha+1) \text { e } I_{i,-1}=0 .
\end{gathered}
$$

(b) Se $x_{i}<x_{i+1} \leq 0$, e $p$ é um ponto arbitrário de $[-1,0]$,

$$
\begin{gathered}
I_{i}=(1-p)^{-\alpha} \sum_{k=0}^{\infty} \frac{\Gamma(k+\alpha)}{\Gamma(\alpha) k !(1-p)^{k}} I_{i, k} \\
I_{i, k}=C\left\{-k(1+p) I_{i, k-1}+\left[\left(1+x_{i+1}\right)^{(1-\beta)}\left(x_{i+1}-p\right)^{k}-\left(1+x_{i}\right)^{(1-\beta)}\left(x_{i}-p\right)^{k}\right]\right\} \\
\text { para } k=0,1,2, \ldots \text { onde } C=1 /(k-\beta+1) \text { e } I_{i,-1}=0 .
\end{gathered}
$$

(c) Se $x_{i} \leq 0 \leq x_{i+1}$, então este intervalo é dividido em dois subintervalos $\left[x_{i}, 0\right]$ e $\left[0, x_{i+1}\right]$ e os itens (a) e (b) são usadas em cada um destes subintervalos. 
Prova: A prova do item (a) pode ser vista em [Gerasoulis 86] ou [Cuminato 87]. Faremos aqui a prova do item (b).

Seja $(1-t)^{-\alpha}$ a parte analítica de $w^{*}(t)$ em $[-1,0]$ e $p$ um ponto arbitrário deste intervalo. Então, podemos escrever:

$$
\begin{aligned}
(1-t)^{-\alpha} & =((1-p)-(t-p))^{-\alpha} \\
& =\sum_{k=o}^{\infty}(-1)^{k}\left(\begin{array}{c}
-\alpha \\
k
\end{array}\right)(1-p)^{-\alpha-k}(t-p)^{k}
\end{aligned}
$$

Substituindo (1.21) em (4.7), temos a expansão em série de Taylor de $(1-t)^{-\alpha}$ em torno do ponto $p$, isto é,

$$
(1-t)^{-\alpha}=(1-p)^{-\alpha} \sum_{k=0}^{\infty} \frac{\Gamma(k+\alpha)}{\Gamma(\alpha) k !(1-p)^{k}}(t-p)^{k}
$$

que converge para todo ponto $p \in[-1,0]$.

Multiplicando esta última equação por $(1+t)^{-\beta}$ e integrando sobre $\left[x_{i}, x_{i+1}\right]$

$$
\begin{aligned}
I_{i} & =\int_{x_{i}}^{x_{i+1}} w^{*}(t) d t \\
& =(1-p)^{-\alpha} \sum_{k=0}^{\infty} \frac{\Gamma(k+\alpha)}{\Gamma(\alpha) k !(1-p)^{k}} I_{i, k}
\end{aligned}
$$

onde

$$
I_{i, k}=\int_{x_{i}}^{x_{i+1}} \frac{(t-p)^{k}}{(1+t)^{\beta}} d t
$$

e provamos assim (4.5).

Para provar (4.6), consideremos a integral indefinida $I_{\bullet, k}$ de $I_{i, k}$ e através da integração por partes, temos

$$
\begin{aligned}
I_{\bullet, k} & =\int \frac{(t-p)^{k}}{(1+t)^{\beta}} d t \\
& =\frac{1}{1-\beta}\left[(t-p)^{k}(1+t)^{1-\beta}-k \int(1+t)^{1-\beta}(t-p)^{k-1} d t\right] \\
& =\frac{1}{1-\beta}\left[(t-p)^{k}(1+t)^{1-\beta}-k \int \frac{((1+p)+(t-p))(t-p)^{k-1}}{(1+t)^{\beta}} d t\right] \\
& =\frac{1}{1-\beta}\left[(t-p)^{k}(1+t)^{1-\beta}-k(1+p) I_{\bullet, k-1}-k I_{\bullet, k}\right]
\end{aligned}
$$

Portanto,

$$
\left(1+\frac{k}{1-\beta}\right) I_{\bullet, k}=\frac{1}{1-\beta}\left[(t-p)^{k}(1+t)^{1-\beta}-k(1+p) I_{\bullet, k-1}\right]
$$


ou

$$
I_{\bullet, k}=\frac{1}{k-\beta+1}\left[(t-p)^{k}(1+t)^{1-\beta}-k(1+p) I_{\bullet, k-1}\right]
$$

e então (4.6) é derivada tomando os limites de integração em (4.10).

É importante notar que como $\alpha, \beta<1$

$$
0 \leq \frac{k(1+p)}{k-\beta+1} \leq 1 \text { se } p \in[-1,0] \text { e } 0 \leq \frac{k(1-p)}{k-\alpha+1} \leq 1 \text { se } p \in[0,1] \text {. }
$$

Assim as recursões (4.4) e (4.6) são numericamente estáveis.

Definição 4.2 Seja $I_{i}$ dada por (4.5) e denotemos por

$$
I_{i}^{(N)}=\sum_{k=0}^{N} d_{i, k}
$$

onde

$$
d_{i, k}=\frac{\Gamma(k+\alpha)}{\Gamma(\alpha) k !(1-p)^{k+\alpha}} I_{i, k}
$$

Definimos $r_{i, N}$ por:

$$
r_{i, N}=I_{i}-I_{i}^{(N)}=\sum_{k=N+1}^{\infty} d_{i, k}
$$

O teorema que vem a seguir dá a velocidade de convergência das somas parciais $I_{i}^{(N)}$ para a série em (4.5).

Teorema 4.2 Se $p=x_{i}$ ou $p=x_{i+1}$, então $r_{i, N}$ das séries em (4.5) satisfaz:

$$
\begin{gathered}
\left|r_{i, N}\right| \leq \frac{\lambda\left|d_{i, N}\right|}{1-\lambda} \leq \frac{\lambda^{N+1-M}\left|d_{i, M}\right|}{1-\lambda} \\
\text { para todo } N \geq M=\left[\frac{1-\alpha}{2}+1\right] \geq 1, \quad \lambda=\frac{x_{i+1}-x_{i}}{1-p}<1
\end{gathered}
$$

onde $[x]$ : denota o maior inteiro contido em $x$. 
Prova: Seja $p=x_{i}$ ou $p=x_{i+1}$.

De (4.9), temos

$$
\begin{aligned}
\left|I_{i, k}\right| & =\left|\int_{x_{i}}^{x_{i+1}} \frac{(t-p)(t-p)^{k-1}}{(1+t)^{\beta}} d t\right| \\
& \leq\left|x_{i+1}-x_{i}\right|\left|\int_{x_{i}}^{x_{i+1}} \frac{(t-p)^{k-1}}{(1+t)^{\beta}} d t\right|
\end{aligned}
$$

ou seja,

$$
\left|I_{i, k}\right| \leq\left|x_{i+1}-x_{i}\right|\left|I_{i, k-1}\right|
$$

Da definição de $d_{i, k}$ dada por (4.11) e da propriedade da função Gama em (1.20), vem

$$
\left|d_{i, k}\right|=\left|\frac{(k+\alpha-1)}{k(1-p)} \frac{\Gamma(k+\alpha-1)}{\Gamma(\alpha)(k-1) !(1-p)^{k+\alpha-1}} I_{i, k}\right|
$$

Usando (4.14), temos

$$
\left|d_{i, k}\right| \leq\left|x_{i+1}-x_{i}\right| \frac{|k+\alpha-1|}{k(1-p)}\left|\frac{\Gamma(k+\alpha-1)}{\Gamma(\alpha)(k-1) !(1-p)^{k+\alpha-1}}\right|\left|I_{i . k-1}\right|
$$

ou

$$
\left|d_{i, k}\right| \leq \frac{|k+\alpha-1|}{k(1-p)}\left|x_{i+1}-x_{i}\right|\left|d_{i, k-1}\right|, k=1,2, \ldots
$$

Como $\alpha<1$ então $|k+\alpha-1|<k$ para todo $k \geq M=\left[\frac{1-\alpha}{2}+1\right] \geq 1$ então de (4.15), segue

$$
\left|d_{i, k}\right| \leq \frac{1}{(1-p)}\left|x_{i+1}-x_{i}\right|\left|d_{i, k-1}\right| \leq \lambda\left|d_{i, k-1}\right|, k=M, M+1, \ldots
$$

onde $\lambda=\left(x_{i+1}-x_{i}\right) /(1-p)<1$ porque $-1 \leq p \leq 0$ e $0<\left(x_{i+1}-x_{i}\right)<1$.

Finalmente, de (4.12) e (4.16), temos

$$
\begin{aligned}
\left|r_{i, N}\right| \leq & \sum_{k=N+1}^{\infty}\left|d_{i, k}\right| \leq \sum_{k=N+1}^{\infty} \lambda^{k-N-1}\left|d_{i, N+1}\right| \\
& \leq \frac{\left|d_{i, N+1}\right|}{1-\lambda} \leq \frac{\lambda^{N+1-M}\left|d_{i, M}\right|}{1-\lambda}, \forall N \geq M .
\end{aligned}
$$

Observe que este teorema implica que a soma parcial $I_{i}^{(N)}$ converge rapidamente para $I_{i}$. Em [Gerasoulis 86] temos um resultado análogo para as séries em (4.3). 


\subsubsection{A quadratura de Gerasoulis}

Teorema 4.3 Seja $p$ um ponto escolhido da seguinte maneira:

$$
p= \begin{cases}x_{i} & \text { se } x>x_{i+1} \\ x_{i+1} & \text { se } x<x_{i}\end{cases}
$$

A integral $L_{i}(x)$ em (4.2) pode ser calculada de acordo com o Caso I, Caso II ou Caso III descritos abaixo.

Caso I: Se $0 \leq x_{i}<x_{i+1}$

então:

$$
L_{i}(x)=\sum_{k=0}^{\infty} B_{k}(x) L_{i, k}(x)
$$

I.A. Cálculo dos $B_{k}(x)$

I.A.1.) $\operatorname{Se} \beta=0$,

$$
B_{k}(x)=\frac{1}{p-x}, k=0,1, \ldots
$$

I.A.2.) $\operatorname{Se} \beta \neq 0$, temos

$$
B_{k}(x)= \begin{cases}0 & k=-1 \\ B_{k-1}(x)+b_{k}(x) & k=0,1, \ldots\end{cases}
$$

com

$$
\begin{gathered}
b_{0}(x)=\frac{(1+p)^{-\beta}}{p-x} \\
b_{k}(x)=-\frac{(k+\beta-1)}{k}\left(\frac{x-p}{1+p}\right) b_{k-1}(x) k=1,2, \ldots
\end{gathered}
$$

I.B.) Cálculo dos $L_{i, k}(x)$

I.B.1.) Se $|x-p| \geq 1$, então :

$$
L_{i, k}(x)=(x-p)^{-k} I_{i, k}(x) \quad k=0,1, \ldots
$$

com $I_{i, k}$ dada por (4.4) . 
I.B.2.) Se $|x-p|<1$, a recursão acima para $L_{i, k}(x)$ deve ser substituida pela recursão equivalente:

$$
\begin{aligned}
& L_{i, k}(x)= \\
= & C\left\{\frac{k(1-p)}{x-p} L_{i, k-1}(x)+\left[\left(1-x_{i+1}\right)^{1-\alpha}\left(\frac{x_{i+1}-p}{x-p}\right)^{k}-\left(1-x_{i}\right)^{1-\alpha}\left(\frac{x_{i}-p}{x-p}\right)^{k}\right]\right\}
\end{aligned}
$$

onde $C=1 /(k-\alpha+1)$ cujo critério de utilização será o seguinte:

I.B.2.a.) Se $|(1-p) /(x-p)|<1$ então $|k(1-p) /((k-\alpha+1)(x-p))|<1$ para $k=0,1,2, \ldots$ Então $L_{i, k}, \quad k=0,1,2, \ldots$ é calculada pela recursão estável $(4.22)$, com $L_{i,-1}(x)=0$.

I.B.2.b.) Se $|(1-p) /(x-p)|>1$ então existe um inteiro $J \geq 1$ para o qual $|k(1-p) /((k-\alpha+1)(x-p))|>1$ para todo $k \geq J$, o que implica que $(4.22)$ será numericamente instável para $k \geq J$.

Para contornar o problema da instabilidade, escolhemos um inteiro $N$ suficientemente grande, $N>J$, fixamos $L_{i, N}(x)=0$, calculamos $L_{i, k}(x)$ para $k=(N-1),(-1), J$ usando (4.22) para trás e $L_{i, k}(x)$, para $k=0,(1),(J-1)$ usando $(4.22)$ para a frente como em (I.B.2.a.).

Caso II: Se $x_{i}<x_{i+1} \leq 0$

então:

$$
L_{i}(x)=\sum_{k=0}^{\infty} B_{k}(x) L_{i, k}(x)
$$

\section{II.A.) Cálculo dos $B_{k}(x)$}

II.A.1.) Se $\alpha=0$

$$
B_{k}(x)=\frac{1}{p-x}, k=0,1,2, \ldots
$$

II.A.2.) Se $\alpha \neq 0$ então:

$$
B_{k}(x)= \begin{cases}0 & k=-1 \\ B_{k-1}(x)+b_{k}(x) & k=0,1, \ldots\end{cases}
$$


com

$$
\begin{gathered}
b_{0}(x)=\frac{(1-p)^{-\alpha}}{p-x} \\
b_{k}(x)=\frac{k+\alpha-1}{k}\left(\frac{x-p}{1-p}\right) b_{k-1}(x), \quad k=1,2, \ldots
\end{gathered}
$$

II.B.) Cálculo dos $L_{i, k}(x)$

II.B.1.) Se $|x-p| \geq 1$ então $L_{i, k}$ é dada por :

$$
L_{i, k}(x)=(x-p)^{-k} I_{i, k}(x) ; k=0,1,2, \ldots
$$

com $I_{i, k}$ dada por (4.6).

II.B.2.) Se $|x-p|<1$, a recursão acima para $L_{i, k}(x)$ deve ser substituida pela recursão equivalente :

$$
\begin{aligned}
& L_{i, k}(x)= \\
= & C\left\{\frac{-k(1+p)}{x-p} L_{i, k-1}(x)+\left[\left(1+x_{i+1}\right)^{1-\beta}\left(\frac{x_{i}-p}{x-p}\right)^{k}-\left(1-x_{i}\right)^{1-\beta}\left(\frac{x_{i}-p}{x-p}\right)^{k}\right]\right\}
\end{aligned}
$$

onde $C=1 /(k-\beta+1)$ cujo critério de utilização é o seguinte:

II.B.2.a.) Se $|(1+p) /(x-p)|<1$ então $|k(1+p) /((k-\beta+1)(x-p))|<1$ para $k=0,1,2 \ldots$ Neste caso, calculamos $L_{i, k}(x)$, para $k=0,1,2, \ldots$, pela recursão numericamente estável $(4.28)$, com $L_{i,-1}(x)=0$.

II.B.2.b) Se $|(1+p) /(x-p)|>1$ então existe um inteiro $J \geq 1$ para o qual $|k(1+p) /((k-\beta+1)(x-p))|>1$ para todo $k \geq J$ e $(4.28)$ será numericamente instável para $k \geq J$. A instabilidade é contornada da mesma maneira que em (I.B.2.b) usando aqui a equação (4.28).

Caso III Se $x_{i}<0<x_{i+1}$

Neste caso o intervalo é dividido em dois subintervalos $\left[x_{i}, 0\right]$ e $\left[0, x_{i+1}\right]$ e os casos I e II são usados para cada um dos subintervalos. 
Prova: A prova do Caso I pode ser vista em [Cuminato 87].

Inicialmente provaremos (4.23).

Seja $p$ como definido no início do teorema (4.3) e $x \notin\left[x_{i}, x_{i+1}\right]$.

Então $|(t-p) /(x-p)|<1$ para $x_{i}<t<x_{i+1} \mathrm{e}$,

$$
(t-x)^{-1}=(p-x)^{-1}\left\{\frac{1}{1-\frac{t-p}{x-p}}\right\}=(p-x)^{-1} \sum_{k=0}^{\infty}\left(\frac{t-p}{x-p}\right)^{k}
$$

Combinando (4.8) e (4.29) segue que

$$
(t-x)^{-1}(1-t)^{-\alpha}=\sum_{k=0}^{\infty} B_{k}(x)\left(\frac{t-p}{x-p}\right)^{k}
$$

com:

$$
B_{k}(x)=(p-x)^{-1}(1-p)^{-\alpha} \sum_{j=0}^{k} \frac{\Gamma(\alpha+j)}{\Gamma(\alpha) j !}\left(\frac{x-p}{1-p}\right)^{j}
$$

Multiplicando ambos os membros da equação (4.30) por $(1+t)^{-\beta}$ e integrando sobre $\left[x_{i}, x_{i+1}\right]$, vem, usando (4.2) que

$$
L_{i}(x)=\sum_{k=0}^{\infty} B_{k}(x) L_{i, k}(x)
$$

onde

$$
L_{i, k}(x)=\int_{x_{i}}^{x_{i+1}}(1+t)^{-\beta}\left(\frac{t-p}{x-p}\right)^{k} d t .
$$

Usando a definição de $B_{k}(x)$ em (4.31), provaremos agora as recursões dadas por (4.25) e (4.26).

Se $\alpha=0$, em (4.29) vê-se claramente que:

$$
B_{k}(x)=\frac{1}{p-x}
$$

para $k=0,1, \ldots$

Se $\alpha \neq 0$, de (4.31) vem

$$
\begin{aligned}
B_{k}(x) & =(p-x)^{-1}(1-p)^{-\alpha}\left[\sum_{j=0}^{k-1} \frac{\Gamma(\alpha+j)}{\Gamma(\alpha) j !}\left(\frac{x-p}{1-p}\right)^{j}+\frac{\Gamma(\alpha+k)}{\Gamma(\alpha) k !}\left(\frac{x-p}{1-p}\right)^{k}\right] \\
& =B_{k-1}(x)+b_{k}(x),
\end{aligned}
$$


onde $b_{k}(x)$ é definido por:

$$
b_{k}(x)=(p-x)^{-1}(1-p)^{-\alpha} \frac{\Gamma(\alpha+k)}{\Gamma(\alpha) k !}\left(\frac{x-p}{1-p}\right)^{k}
$$

e satisfaz a relação de recorrência (4.26).

\section{Estabilidade dos $B_{k}(x)$}

A estabilidade dos $B_{k}(x)$, obviamente depende da estabilidade dos $b_{k}(x)$. Como $\alpha<1$, então $|k+\alpha-1|<k$ para todo $k>M=[(1-\alpha) / 2+1]$. Se

$$
\left|\frac{x-p}{1-p}\right|<1 \quad \text { então } \quad\left|\frac{(k+\alpha-1)(x-p)}{k(1-p)}\right|<1
$$

e a recursão (4.26) é numericamente estável.

Por outro lado, se

$$
\left|\frac{x-p}{1-p}\right| \geq 1
$$

deve existir um inteiro $J$, tal que

$$
\left|\frac{(k+\alpha-1)(x-p)}{k(1-p)}\right| \geq 1
$$

para todo $k>J$. Então, para $k=J, J+1, \ldots$, a recursão (4.26) será numericamente instável e deve ser substituida pela recursão equivalente:

$$
\frac{1}{b_{k}(x)}=\frac{k(1-p)}{(k+\alpha-1)(x-p)} \frac{1}{b_{k-1}}
$$

enquanto que, para $k=(J-1),(-1), 1$ a recursão (4.26) será numericamente estável.

Em outras palavras, $b_{J}(x), b_{J+1}(x), \ldots$ serão calculados por $(4.35)$ e $b_{J-1}(x), \ldots, b_{1}(x)$ por (4.26).

Finalmente, a relação de recorrência (4.27) envolvendo $L_{i, k}(x)$ segue do uso da definição dada por (4.33). De fato,

$$
\begin{aligned}
L_{i, k}(x) & =(x-p)^{-k} \int_{x_{i}}^{x_{i+1}}(1+t)^{-\beta}(t-p)^{k} d t \\
& =(x-p)^{-k} I_{i, k}
\end{aligned}
$$

com $I_{i, k}$ dado por (4.6). 
A equação (4.28) é obtida fazendo a divisão da equação (4.6) por $(x-p)^{-k}$.

A separação de (4.28) em II.B.2.a) e II.B.2.b) tem o propósito de obter a estabilidade numérica da relação de recorrência.

De acordo com o exposto em [Cuminato 87] podemos calcular todos os $L_{i, k}(x)$ dentro de uma tolerância $\epsilon$, bastando para isto, escolher um inteiro $N$ de acordo com a desigualdade que será derivada abaixo, fazendo uso de (4.33), (4.9) e (4.14),

$$
\begin{aligned}
\left|L_{i, N}(x)\right| & =\left|\int_{x_{i}}^{x_{i+1}}(1+t)^{-\beta}\left(\frac{t-p}{x-p}\right)^{N} d t\right| \\
& =\left|(x-p)^{-N} \int_{x_{i}}^{x_{i+1}}(1+t)^{-\beta}(t-p)^{N} d t\right| \\
& \leq\left[\frac{\left|x_{i+1}-x_{i}\right|}{|x-p|}\right]^{N}\left|I_{i, 0}\right|<\epsilon,
\end{aligned}
$$

resultando para $N$ a desigualdade:

$$
N \geq \frac{\log \left|\epsilon / I_{i, 0}\right|}{\log \left(\lambda_{2}\right)}, \quad \text { onde } \quad \lambda_{2}=\frac{\left|x_{i+1}-x_{i}\right|}{|x-p|}
$$

\section{Erro de Truncamento}

Vamos agora analisar o erro na aproximação numérica de $L_{i}(x)$ pelo truncamento da série (4.23) no $m$-ésimo termo.

Usaremos aqui a seguinte notação:

$$
\begin{gathered}
L_{i}^{(m)}(x)=\sum_{k=0}^{m} B_{k}(x) L_{i, k}(x) \\
R_{i}^{(m)}(x)=L_{i}(x)-L_{i}^{m}(x)=\sum_{k=m+1}^{\infty} L_{i}^{(m)}(x)
\end{gathered}
$$

Teorema 4.4 Se $x_{i}<x_{i+1} \leq 0$ então o resto $R_{i}^{(m)}$ satisfaz

$$
\left|R_{i}^{(m)}(x)\right| \leq C(x)\left|I_{i, 0}\right| \lambda_{2}^{m+1}
$$

para todo $m \geq M=\left[\frac{1-\alpha}{2}+1\right] \geq 1$, onde

$$
C(x)=\frac{\left|B_{\infty}(x)\right|}{1-\lambda_{2}}+\frac{\left|b_{M}(x)\right| \lambda_{1}^{2}}{(1-\lambda)\left(1-\lambda_{1}\right)}
$$


é independente de $m e$,

$$
\begin{aligned}
B_{\infty}(x) & =\frac{(1-x)^{-\alpha}}{p-x}, \\
\lambda_{1} & =\frac{|x-p|}{1-p}<1, \\
\lambda_{2} & =\frac{\left|x_{i+1}-x_{i}\right|}{|x-p|}<1, \\
\lambda & =\lambda_{1} \lambda_{2}<1,
\end{aligned}
$$

Prova: Da escolha de $M$ e do fato que $\alpha<1$ temos $|j+\alpha-1|<j$ para todo $j \geq M$. Da relação de recorrência (4.26), obtemos

$$
\left|b_{j}(x)\right| \leq\left|\frac{x-p}{1-p}\right| \quad\left|b_{j-1}(x)\right|
$$

ou

$$
\left|b_{j}(x)\right| \leq \lambda_{1}^{j-k}\left|b_{k}(x)\right|, j=k, k+1, \ldots \text { onde } k \geq M
$$

Da definição de $b_{k}(x)$ em (4.34) vem que

$$
\sum_{k=0}^{\infty} b_{k}(x)=(p-x)^{-1}(1-p)^{-\alpha} \sum_{k=0}^{\infty} \frac{\Gamma(\alpha+k)}{\Gamma(\alpha) k !(1-p)^{k}}(x-p)^{k} .
$$

Comparando a expressão acima com (4.8)obtemos

$$
\sum_{k=0}^{\infty} b_{k}(x)=(p-x)^{-1}(1-x)^{-\alpha}=B_{\infty}(x)
$$

De (4.31), vemos que $B_{k}(x)$ também pode ser dado por:

$$
B_{k}(x)=\sum_{j=0}^{k} b_{j}(x)=B_{\infty}(x)-\sum_{j=k+1}^{\infty} b_{j}(x)
$$

Portanto,

$$
\left|B_{k}(x)\right| \leq\left|B_{\infty}(x)\right|+\sum_{j=k+1}^{\infty}\left|b_{j}(x)\right|
$$


e de (4.42), obtemos

$$
\begin{gathered}
\left|B_{k}(x)\right| \leq\left|B_{\infty}(x)\right|+\sum_{j=k+1}^{\infty} \lambda_{1}^{j-k-1}\left|b_{k+1}(x)\right| \\
\leq\left|B_{\infty}(x)\right|+\left|b_{k+1}(x)\right| \sum_{j=0}^{\infty} \lambda_{1}^{j}
\end{gathered}
$$

e portanto

$$
\left|B_{k}(x)\right| \leq\left|B_{\infty}(x)\right|+\frac{\left|b_{k+1}(x)\right|}{1-\lambda_{1}}
$$

A função $f(t)=(1+t)^{-\beta}\left(\frac{t-p}{x-p}\right)^{k}$ não muda de sinal para $t \in\left[x_{i}, x_{i+1}\right]$, então

$$
\left|\int_{x_{i}}^{x_{i+1}} f(t) d t\right|=\int_{x_{i}}^{x_{i+1}}|f(t)| d t
$$

Usando este fato

$$
\begin{aligned}
\left|L_{i, k}(x)\right| & =\left|\int_{x_{i}}^{x_{i+1}}(1+t)^{-\beta}\left(\frac{t-p}{x-p}\right)^{k} d t\right| \\
& =\int_{x_{i}}^{x_{i+1}}\left|\frac{t-p}{x-p}\right|\left|(1+t)^{-\beta}\left(\frac{t-p}{x-p}\right)^{k-1}\right| d t \\
& \leq \frac{\left|x_{i+1}-x_{i}\right|}{|x-p|} \int_{x_{i}}^{x_{i+1}}\left|(1+t)^{-\beta}\left(\frac{t-p}{x-p}\right)^{k-1}\right| d t \\
& =\lambda_{2}\left|\int_{x_{i}}^{x_{i+1}}(1+t)^{-\beta}\left(\frac{t-p}{x-p}\right)^{k-1} d t\right|=\lambda_{2}\left|L_{i, k-1}(x)\right|
\end{aligned}
$$

ou

$$
\left|L_{i, k}(x)\right| \leq \lambda_{2}^{k-v}\left|L_{i, v}(x)\right|, k=v, v+1, \ldots ; v \geq 0
$$

De (4.37) e (4.38), temos

$$
\left|R_{i}^{(m)}(x)\right| \leq \sum_{k=m+1}^{\infty}\left|B_{k}(x)\right|\left|L_{i, k}(x)\right|
$$

Usando (4.43), (4.44) e (4.42), nesta ordem, temos

$$
\left|R_{i}^{(m)}(x)\right| \leq \sum_{k=m+1}^{\infty}\left[\left|B_{\infty}(x)\right|+\frac{\left|b_{k+1}(x)\right|}{1-\lambda_{1}}\right] \lambda_{2}^{k-m-1}\left|L_{i, m+1}(x)\right|
$$




$$
\begin{aligned}
& \leq \sum_{k=m+1}^{\infty}\left[\left|B_{\infty}(x)\right|+\lambda_{1}^{k-m} \frac{\left|b_{m+1}(x)\right|}{1-\lambda_{1}}\right] \lambda_{2}^{k-m-1}\left|L_{i, m+1}(x)\right| \\
& \leq\left|L_{i, m+1}(x)\right|\left\{\left|B_{\infty}(x)\right| \sum_{k=m+1}^{\infty} \lambda_{2}^{k-m-1}+\frac{\left|b_{m+1}(x)\right| \lambda_{1}}{\left(1-\lambda_{1}\right)} \sum_{k=m+1}^{\infty}\left(\lambda_{1} \lambda_{2}\right)^{k-m-1}\right\} .
\end{aligned}
$$

Finalmente,

$$
\left|R_{i}^{(m)}(x) \leq\right| L_{i, m+1}(x) \mid\left\{\frac{\left|B_{\infty}(x)\right|}{1-\lambda_{2}}+\frac{\left|b_{m+1}(x)\right| \lambda_{1}}{\left(1-\lambda_{1}\right)(1-\lambda)}\right\},
$$

onde $\lambda=\lambda_{1} \lambda_{2}$.

Usando (4.42) e (4.44), podemos escrever

$$
\begin{gathered}
\left|b_{m+1}(x)\right| \leq \lambda_{1}^{m+1-M}\left|b_{M}(x)\right| \leq \lambda_{1}\left|b_{M}(x)\right| \\
\left|L_{i, m+1}(x)\right| \leq \lambda_{2}^{m+1}\left|L_{i, 0}(x)\right|=\lambda_{2}^{m+1}\left|I_{i, 0}\right|
\end{gathered}
$$

Substituindo estas duas expressões em (4.45) temos o resultado final como em (4.39) e (4.40).

\section{Comentários}

1. Em [Gerasoulis 86] ou [Cuminato 87] pode ser encontrado um resultado semelhante para o caso $0 \leq x_{i}<x_{i+1}$.

2. Dado $\epsilon>0$ podemos encontrar $m$ tal que

$$
\left|R_{i}^{(m)}\right|<\epsilon \text { fazendo } C(x)\left|I_{i, 0}\right| \lambda_{2}^{m+1}<\epsilon
$$

Portanto, qualquer inteiro $m$ satisfazendo

$$
m>\log \left(\left|\frac{\epsilon}{C(x) I_{i, 0}}\right|\right) / \log \lambda_{2}
$$

produz o erro com a tolerância dada.

3. Para $x$ e $\epsilon$ dados, os valores de $C(x), I_{i, 0}$ e $\lambda_{2}$ são conhecidos.

4. Avaliar $L_{i}(x)$ com $x$ perto de qualquer dos extremos de integração tem um custo computacional extremamente grande. Se $m$ é o número de termos da soma na quadratura de $L_{i}(x)$, este número torna-se muito grande quando $\lambda_{2}$ tende a 1 . Isto acontece quando $x$ se aproxima de $x_{i}$ ou $x_{i+1}$. 


\subsection{Aplicações numéricas}

Consideremos o problema de resolver a integral

$$
r(x)=\int_{-1}^{0} \frac{w^{*}(t)}{t-x} d t, x \notin(-1,0), w^{*}(t)=(1-t)^{-1 / 2}(1+t)^{-1 / 2} .
$$

pela quadratura de Gerasoulis.

Dados $x$ e $\epsilon$, a tabela (4.1) mostra o número de termos $m$ necessários para que o resto $R_{i}^{(m)}(x)$ satisfaça

$$
\left|R_{i}^{(m)}(x)\right| \leq \epsilon
$$

\begin{tabular}{|c|c|c|}
\hline$x$ & $1.0 \mathrm{E}-5$ & $1.0 \mathrm{E}-10$ \\
\hline 0.5 & 31 & 59 \\
\hline $1.0 \mathrm{~d}-1$ & 142 & 263 \\
\hline $1.0 \mathrm{~d}-3$ & 18026 & 29545 \\
\hline $1.0 \mathrm{~d}-5$ & 2287249 & 3450827 \\
\hline $1.0 \mathrm{~d}-7$ & 273242695 & 394371898 \\
\hline
\end{tabular}

quando, por exemplo, $\mathrm{x}$ tende a $0^{+}$.

Tabela 4.1: Número de termos da quadratura de Gerasoulis

Os números da tabela (4.1) mostram que é necessário um algoritmo melhor para calcular a integral quando $x$ se aproxima de zero.

De fato, da definição de $\lambda_{2}$ em (4.41) temos que $\lambda_{2}$ tende a 1 , quando $x \rightarrow x_{i+1}$ ou $x \rightarrow x_{i}$ e conseqüentemente de $(4.46), m$ tende ao infinito. Assim, calcular $L_{i}(x)$ pelo truncamento da série no $m$-ésimo termo, vai representar um enorme esforço computacional podendo até tornar inviável o uso, na prática, da quadratura em questão.

Para resolver este problema devemos reduzir o tamanho do intervalo de integração. Isto feito teremos um valor menor para $\lambda_{2}$ e de (4.46) também para $m$.

Descrevemos a seguir um algoritmo que utiliza esta idéia para diminuir o número de termos somados na série. 


\subsection{Algoritmo do Ponto Médio (A.P.M.)}

Sejam $L_{i}(x)$ como em $(4.2)$ e $\bar{x} \in\left[x_{i}, x_{i+1}\right]$. Então,

$$
L_{i}(x)=\int_{x_{i}}^{\bar{x}} \frac{w^{*}(t)}{t-x} d t+\int_{\bar{x}}^{x_{i+1}} \frac{w^{*}(t)}{t-x} d t
$$

É óbvio que o valor de $\lambda_{2}$ na primeira integral da equação acima é menor que em (4.2) e neste caso esta integral será calculada com um número muito menor de termos. Este ponto $\bar{x}$ pode ser escolhido convenientemente de modo que o número de termos necessários para se obter um erro menor do que $\epsilon$ não exceda um número predeterminado $m_{0}$.

O algoritmo do ponto médio (A.P.M.), como o próprio nome já sugere, escolhe para $\bar{x}$ o ponto médio do intervalo $\left[x_{i}, x_{i+1}\right]$,ou seja,

$$
\bar{x}=\frac{\left(x_{i+1}+x_{i}\right)}{2} \text {. }
$$

Para a segunda integral de (4.48), repetimos a divisão do intervalo [ $\left.\bar{x}, x_{i+1}\right]$, tendo em mente a mesma precisão $\epsilon$ e o mesmo número de termos $m_{0}$. Assim,

$$
L_{i}(x)=\sum_{j=1}^{s} \int_{x_{i j}}^{x_{i j+1}} \frac{w^{*}(t)}{t-x} d t \text { onde } x_{i}=x_{i 1}<x_{i 2}<\ldots<x_{i s}=x_{i+1}
$$

Melhores resultados serão conseguidos se o número $s$ não for muito grande, pois caso contrário, embora todas as integrais sejam calculadas com erro menor que $\epsilon$ não podemos garantir a mesma precisão no resultado final.

Veja abaixo, os passos a serem seguidos para resolver a integral dada por (4.47) usando o Algoritmo do Ponto Médio:

1) Faça $x_{i}=-1, x_{i+1}=0$.

2) Calcule $m$ em (4.46).

3) Se $m<m_{0}$ então 6).

4) Faça $x_{i}=x_{i}, x_{i+1}=1 / 2\left(x_{i}+x_{i+1}\right)$.

5) Repita o passo 2).

6) Calcule $L_{i}(x)$ pelo teorema 4.3, Caso II.

7) $r(x)=r(x)+L_{i}(x)$ 
8) Se $x_{i+1}=0$ então 10$)$

9) Faça $x_{i}=x_{i+1}, x_{i+1}=x_{0}$ e repita o passo 2)

10)Fim

A tabela (4.2) nos mostra agora o número total de termos usados em (4.37) de modo a tornar o erro menor que $\epsilon$, para $x$ tendendo a $x_{i+1}, \operatorname{com} m_{0}=50$.

\begin{tabular}{|c|c|c|}
\hline$x$ & $1.0 \mathrm{E}-05$ & $1.0 \mathrm{E}-10$ \\
\hline 0.5 & 31 & 52 \\
\hline $1.0 \mathrm{~d}-1$ & 55 & 114 \\
\hline $1.0 \mathrm{~d}-3$ & 162 & 325 \\
\hline $1.0 \mathrm{~d}-5$ & 283 & 539 \\
\hline $1.0 \mathrm{~d}-7$ & 381 & 773 \\
\hline
\end{tabular}

Tabela 4.2: Número de termos usando A.P.M.

Comparando as duas tabelas (4.1) e (4.2) podemos observar que o uso de $\bar{x}$ como ponto médio trouxe uma melhora extraordinária no algoritmo do teorema 4.3.

A integral (4.47), resolvida pela quadratura de Gerasoulis associado ao algoritmo do ponto médio usando $\epsilon=1.0 d-10$ e $m_{0}=50$, é dada pela tabela (4.3). .

\begin{tabular}{||l|l|l||}
\hline$x$ & $f_{-1}^{0} w^{*}(t)(t-x)^{-1} d t$ & $\mid$ Erro $\mid$ \\
\hline 0.5 & -1.520691992601893 & $2.5 \mathrm{E}-12$ \\
\hline $1.0 \mathrm{~d}-1$ & -3.008302149788701 & $6.6 \mathrm{E}-11$ \\
\hline $1.0 \mathrm{~d}-3$ & -7.600905992124754 & $1.7 \mathrm{E}-08$ \\
\hline $1.0 \mathrm{~d}-5$ & -12.206071193939750 & $1.4 \mathrm{E}-06$ \\
\hline $1.0 \mathrm{~d}-6$ & -14.508627146057010 & $3.0 \mathrm{E}-05$ \\
\hline $1.0 \mathrm{~d}-7$ & -16.810884121997730 & $3.5 \mathrm{E}-04$ \\
\hline $1.0 \mathrm{~d}-8$ & -1.414213548230960 & 17.69961 \\
\hline
\end{tabular}

Tabela 4.3: Valor da integral usando o A.P.M.

A análise desta tabela nos mostra que quanto mais $x$ se aproxima de um dos extremos de integração, maior é o valor absoluto do erro e o resultado pode ser até incorreto como no caso, de $x=1.0 d-8$. Isto ocorre porque, sendo o número de termos da quadratura muito grande, muitas subdivisões serão feitas até que se consiga resolver a integral com $m<m_{0}=50$ termos. 
Resolvemos este problema,neste trabalho, diminuindo ao máximo o número de subdivisões e para isto escolhemos $\bar{x}$ tal que o intervalo $\left[x_{i}, \bar{x}\right]$ seja o maior possível para o qual a integral seja calculada com erro menor que $\epsilon$,usando $m_{0}$ termos nas séries.

\section{Obtenção de $\bar{x}$}

Vamos agora obter o valor de $\bar{x}$ no cálculo de

$$
\int_{x_{i}}^{\bar{x}} \frac{w^{*}(t)}{t-x} d t
$$

no caso em que $x$ está muito próximo de $x_{i}$.

Da definição de $\lambda_{2}$ em (4.41) e tomando $p$ de acordo com o disposto anteriormente, ou seja, $p=\bar{x}$ vem

$$
\lambda_{2}=\frac{\left|\bar{x}-x_{i}\right|}{|x-\bar{x}|} .
$$

Assim

$$
\begin{aligned}
\bar{x}-x_{i} & =\lambda_{2}(\bar{x}-x) \\
\bar{x}-\lambda_{2} \bar{x} & =x_{i}-\lambda_{2} x .
\end{aligned}
$$

Portanto

$$
\bar{x}=\frac{x_{i}-\lambda_{2} x}{1-\lambda_{2}}
$$

onde $\lambda_{2}$ é raiz da equação

$$
f\left(\lambda_{2}\right)=\lambda_{2}^{m+1}\left|I_{i, 0}\right|\left[\frac{\left|B_{\infty}(x)\right|}{1-\lambda_{2}}+\frac{\left|b_{M}(x)\right| \lambda_{1}^{2}}{(1-\lambda)\left(1-\lambda_{1}\right)}\right]-\epsilon=0
$$

podendo ser obtida, por exemplo, pelo método numérico da bissecção.

As equações (4.50) e (4.51) serão usadas no próximo capítulo.

\subsection{Quadratura para $S_{i}(x)$}

Desenvolvemos aqui, para ser usada posteriormente, a avaliação numérica da integral

$$
S_{i}(x)=\int_{x_{i}}^{x_{i+1}} \frac{w^{*}(t)-w^{*}(x)}{t-x} d t
$$


onde $x_{i}, x_{i+1} \in(-1,1) ; w^{*}(x)=(1-x)^{-\alpha}(1+x)^{-\beta}$ e $x$ está muito próximo de um dos extremos de integração $x_{i}$ ou $x_{i+1}$.

Usando a expansão de $w^{*}(t)$ em torno do ponto $x$, da maneira como foi proposta em [Miller 85]

$$
w^{*}(t)=(1-t)^{-\alpha}(1+t)^{-\beta}=\sum_{n=0}^{\infty} A_{n}(x)(t-x)^{n}
$$

onde

$$
A_{n}(x)=(1-x)^{-\alpha}(1+x)^{-\beta} \sum_{j=0}^{n} \frac{(\alpha)_{j}(\beta)_{n-j}(-1)^{n-j}}{j !(n-j) !(1-x)^{j}(1+x)^{n-j}}
$$

e

$$
(\alpha)_{j}=\frac{\Gamma(j+\alpha)}{\Gamma(\alpha)}
$$

podemos deduzir o seguinte

$$
\begin{aligned}
S_{i}(x) & =\int_{x_{i}}^{x_{i+1}} \frac{w^{*}(t)-w^{*}(x)}{t-x} d t \\
& =\int_{x_{i}}^{x_{i+1}} \frac{\sum_{n=0}^{\infty} A_{n}(x)(t-x)^{n}-w^{*}(x)}{t-x} d t \\
& =\int_{x_{i}}^{x_{i+1}}\left[\sum_{n=1}^{\infty} \frac{A_{n}(x)(t-x)^{n}}{t-x}+\frac{(1-x)^{-\alpha}(1+x)^{-\beta}-w^{*}(x)}{t-x}\right] d t \\
& =\sum_{n=1}^{\infty} A_{n}(x) \int_{x_{i}}^{x_{i+1}}(t-x)^{n-1} d t \\
& \left.=\sum_{n=1}^{\infty} \frac{A_{n}(x)}{n}(t-x)^{n}\right]_{x_{i}}^{x_{i+1}} .
\end{aligned}
$$

Assim

$$
S_{i}(x)=\sum_{n=1}^{\infty} \frac{A_{n}(x)}{n}\left[\left(x_{i+1}-x\right)^{n}-\left(x_{i}-x\right)^{n}\right]
$$

Comprovamos na prática que a quadratura dada por (4.55) converge muito rapidamente. Os $A_{n}(x)$ geralmente assumem valores elevados, mas, em se realizando as operações indicadas dentro dos colchetes iremos obter valores muito pequenos, fazendo 
com que no geral cada termo desta série tenda a zero. O truncamento desta série é feito observando a diferença entre um termo e o seu anterior de acordo com uma tolerância predeterminada $\delta$.

Devemos salientar que, de (4.54), para $x$ muito próximo de 1 ou de -1 , esta quadratura não pode ser aplicada. 


\section{Capítulo 5}

\section{Equação Integral Singular com Coeficientes Constantes e Dados não Suaves}

\subsection{Introdução}

Neste capítulo apresentamos a teoria das Equações Integrais Singulares com Coeficientes Constantes, tendo no seu lado direito a função de Heaviside.

Comparando esta teoria com aquela estudada no Capítulo 3 , analisamos na seção 5.2 , o comportamento da solução exata.

A função $f(x)$ sendo agora descontínua não satisfaz a condição de Hölder em $[-1,1]$. Assim a convergência da solução numérica para a solução exata, antes garantida pelo teorema 3.1 , já não está mais assegurada.

Para que possamos aplicar o método de colocação polinomial, obviamente com a garantia de convergência, necessário se torna a utilização de uma estratégia, descrita no início da seção 5.3 , que torne o lado direito da equação integral mais regular.

Apresentamos a seguir o método de resolução da equação integral que é o objetivo deste trabalho, usando resultados dos capítulos anteriores já existentes ou demonstrados para este fim.

Finalmente, em exemplos numéricos mostramos a eficiência do método proposto. 


\subsection{Comportamento da Solução Exata da Equação Integral}

Seja a equação integral

$$
a \phi(x)+\frac{b}{\pi} f_{-1}^{1} \frac{\phi(t)}{t-x} d t+\int_{-1}^{1} k(x, t) \phi(t) d t=f(x), x \in[-1,1] .
$$

onde os coeficientes $a$ e $b$ são constantes reais, $k(x, t)$ é Hölder contínua e $f(x)$ é a função de Heaviside definida por:

$$
f(x)=\left\{\begin{array}{cc}
0 & -1 \leq x<x_{0} \\
1 & x_{0}<x \leq 1
\end{array} \quad x_{0} \in(-1,1) .\right.
$$

A descontinuidade de $f(x)$ não impede que à equação (5.1) possa ser aplicada a teoria do Capítulo 3. Este resultado pode ser encontrados em [Gakhov 66] e [Khvedelidze 56].

Dependendo da classe de funções onde a equação for resolvida, (5.1) pode ter infinitas soluções ou não ter nenhuma solução, e esta caso exista, pode apresentar singularidades nos extremos -1 e 1 . Este fato teve sua explicação no Capítulo 3 e as possíveis classes de soluções são as mesmas descritas lá.

Do que foi dito acima, as funções $G(x), \Gamma(x), \Pi(x), Z(x), w(x)$, os números $\alpha$, $\beta$ e finalmente, o índice $\kappa$, para (5.1) são definidos exatamente da mesma maneira que para (3.1).

Um outro resultado importante é que a solução da equação (5.1) pode ainda ser decomposta na forma dada por (2.13), mas $g$ não será Hölder contínua em [-1,1]. Usando a notação de operadores, como no Capítulo 3 , a equação (5.1) pode ser escrita na forma

$$
H g+K g=f \text {. }
$$

Aplicando à (5.2) o operador $H^{I}$, vem

$$
g+H^{I} K g=H^{I} f+g_{0},
$$

onde $g_{0}$ pertencente ao núcleo de $H$ e o operador $H^{I}$ é dado pela fórmula (3.8).

Do teorema $1.3 \mathrm{em}(3.8)$ temos que a singularidade de $f$. no ponto $x_{0}$ resulta numa singularidade logaritmica em $H^{I}$.f no referido ponto. Da singularidade de $H^{I} f$ em $x_{0}$, o teorema 1.3 garante para (5.3), uma singularidade de $g(x)$ em $x_{0}$. A equação (5.3) é uma equação de Fredholm. 


\subsection{Solução Numérica da Equação Integral}

Uma vez conhecido o comportamento da solução exata de (5.1) foi proposta em [Cuminato 87] uma estratégia para a sua solução numérica. Vamos descrevê-la aqui.

Tomemos (5.2). Suponhamos que exista uma função $u$ tal que

$$
H u=f \text {, onde } f \text { é a função de Heaviside }
$$

Então, a solução $g$ de (5.2) pode ser escrita como

$$
g=u+v
$$

Assim, de

$$
H g+K g=f
$$

temos:

$$
\begin{aligned}
& H(u+v)+K(u+v)=f \\
& H u+H v+K u+K v=f
\end{aligned}
$$

Usando (5.4) temos a seguinte expressão para $v$ :

$$
H v+K v=-K u
$$

Uma característica importante de (5.6), é que o seu lado direito é muito mais suave do que na equação original.

Antes de pensarmos em um método numérico para resolver (5.6), devemos obter uma expressão para $u$ e a seguir calcular $-K u$.

\subsubsection{Avaliação numérica da função $u$}

Em [Cuminato 87] podemos ver que se $u$ é a função que satisfaz (5.4), então

$$
u(x)=H^{I} f(x)=a w^{*}(x) f(x)-\frac{b}{\pi}\left[f_{-1}^{x_{0}} \frac{w^{*}(t) f(t)}{t-x} d t+f_{x_{0}}^{1} \frac{w^{*}(t) f(t)}{t-x} d t\right] .
$$

Mas $f$ é a função de Heaviside e assim sendo $u(x)$ pode ser dada por:

$$
u(x)=a w^{*}(x) f(x)-\frac{b}{\pi} f_{x_{0}}^{1} \frac{w^{*}(t)}{t-x} d t
$$


Vemos assim que a avaliação numérica de $u(x)$ se reduz ao cálculo aproximado da integral singular presente em (5.7).

$$
\begin{aligned}
\text { Para } x \notin\left[x_{0}, 1\right], f(x)= & 0, \\
& u(x)=\frac{-b}{\pi} f_{x_{0}}^{1} \frac{w^{*}(t)}{t-x} d t
\end{aligned}
$$

e esta integral pode ser aproximada pela fórmula de quadratura de Gerasoulis, vista no Capítulo 4.

Vamos mostrar a seguir como fica a expressão para $u(x)$ quando $x \in\left(x_{0}, 1\right)$.

Para $x \in\left(x_{0}, 1\right), f(x)=1$. De $(5.7)$, vem

$$
\begin{aligned}
u(x) & =a w^{*}(x)-\frac{b}{\pi} f_{x_{0}}^{1} \frac{w^{*}(t)}{t-x} d t \\
& =a w^{*}(x)-\frac{b}{\pi}\left[f_{-1}^{1} \frac{w^{*}(t)}{t-x} d t-f_{-1}^{x_{0}} \frac{w^{*}(t)}{t-x} d t\right]
\end{aligned}
$$

Usando (3.13),

$$
u(x)=-\frac{b 2^{\kappa}}{\sin \pi \alpha} P_{\kappa}^{(-\alpha,-\beta)}(x)+\frac{b}{\pi} f_{-1}^{x_{0}} \frac{w^{*}(t)}{t-x} d t
$$

onde $\kappa=-(\alpha+\beta)$ e $P_{\kappa}^{(-\alpha,-\beta)}(x)$ é o polinômio de Jacobi de grau $\kappa$.

Resumindo, $u(x)$ fica então definida da seguinte maneira:

$$
\begin{array}{cc}
u(x)=\frac{-b}{\pi} f_{x_{0}}^{1} \frac{w^{*}(t)}{t-x} d t & x \notin\left(x_{0}, 1\right) \\
u(x)=\frac{-b 2^{\kappa}}{\sin \pi \alpha} P_{\kappa}^{(-\alpha,-\beta)}(x)+\frac{b}{\pi} f_{-1}^{x_{0}} \frac{w^{*}(t)}{t-x} d t & x \in\left(x_{0}, 1\right)
\end{array}
$$

Observe que como $u(x)$ é singular em $x_{0}$, este ponto foi excluido do domínio de $u$.

\subsubsection{Avaliação numérica da função $K u$}

Seja

$$
K u(x)=\int_{-1}^{1} w(t) k(x, t) u(t) d t
$$


Para $x_{0} \in[-1,1]$ temos

$$
K u(x)=\int_{-1}^{x 0} w(t) k(x, t) u(t) d t+\int_{x 0}^{1} w(t) k(x, t) u(t) d t
$$

Substituindo (5.9) e (5.10) em (5.11), obtemos

$$
\begin{aligned}
K u(x)= & \int_{-1}^{x 0} w(t) k(x, t)\left[\frac{-b}{\pi} f_{x_{0}}^{1} \frac{w^{*}(s)}{s-t} d s\right] d t+\int_{x 0}^{1} w(t) k(x, t)\left[\frac{-b 2^{\kappa}}{\sin \pi \alpha} P_{\kappa}^{(-\alpha,-\beta)}(t)+\right. \\
& \left.+\frac{b}{\pi} f_{-1}^{x_{0}} \frac{w^{*}(s)}{s-t} d s\right] d t= \\
= & \frac{-b 2^{\kappa}}{\sin \pi \alpha} \int_{x 0}^{1} w(t) k(x, t) P_{\kappa}^{(-\alpha,-\beta)}(t) d t-\frac{b}{\pi} \int_{-1}^{x 0} w(t) k(x, t) f_{x_{0}}^{1} \frac{w^{*}(s)}{s-t} d s d t+ \\
& +\frac{b}{\pi} \int_{x 0}^{1} w(t) k(x, t) f_{-1}^{x_{0}} \frac{w^{*}(s)}{s-t} d s d t
\end{aligned}
$$

Adicionando e subtraindo $w^{*}(t)$ nos integrandos das duas últimas integrais acima, temos

$$
\begin{aligned}
K u(x)= & \frac{-b 2^{\kappa}}{\sin \pi \alpha} \int_{x 0}^{1} w(t) k(x, t) P_{\kappa}^{(-\alpha,-\beta)}(t) d t \\
& -\frac{b}{\pi} \int_{-1}^{x 0} w(t) k(x, t) f_{x_{0}}^{1} \frac{w^{*}(t)+w^{*}(s)-w^{*}(t)}{s-t} d s d t+ \\
& +\frac{b}{\pi} \int_{x 0}^{1} w(t) k(x, t) f_{-1}^{x_{0}} \frac{w^{*}(t)+w^{*}(s)-w^{*}(t)}{s-t} d s d t= \\
= & \frac{-b 2^{\kappa}}{\sin \pi \alpha} \int_{x 0}^{1} w(t) k(x, t) P_{\kappa}^{(-\alpha,-\beta)}(t) d t-\frac{b}{\pi} \int_{-1}^{x 0} w(t) k(x, t) w^{*}(t) f_{x_{0}}^{1} \frac{d s}{s-t} d t+
\end{aligned}
$$




$$
\begin{aligned}
& -\frac{b}{\pi} \int_{-1}^{x 0} w(t) k(x, t) f_{x_{0}}^{1} \frac{w^{*}(s)-w^{*}(t)}{s-t} d s d t+\frac{b}{\pi} \int_{x 0}^{1} w(t) k(x, t) w^{*}(t) f_{-1}^{x_{0}} \frac{d s}{s-t} d t+ \\
& +\frac{b}{\pi} \int_{x 0}^{1} w(t) k(x, t) f_{-1}^{x_{0}} \frac{w^{*}(s)-w^{*}(t)}{s-t} d s d t= \\
& =\frac{-b 2^{\kappa}}{\sin \pi \alpha} \int_{x 0}^{1} w(t) k(x, t) P_{\kappa}^{(-\alpha,-\beta)}(t) d t-\frac{b}{\pi} \int_{-1}^{x_{0}} k(x, t) \ln \left|\frac{1-t}{x_{0}-t}\right| d t+ \\
& -\frac{b}{\pi} \int_{-1}^{x 0} w(t) k(x, t) f_{x_{0}}^{1} \frac{w^{*}(s)-w^{*}(t)}{s-t} d s d t+\frac{b}{\pi} \int_{x_{0}}^{1} k(x, t) \ln \left|\frac{x_{0}-t}{1+t}\right| d t+ \\
& +\frac{b}{\pi} \int_{x 0}^{1} w(t) k(x, t) f_{-1}^{x_{0}} \frac{w^{*}(s)-w^{*}(t)}{s-t} d s d t= \\
& =\frac{-b 2^{\kappa}}{\sin \pi \alpha} \int_{x 0}^{1} w(t) k(x, t) P_{\kappa}^{(-\alpha,-\beta)}(t) d t-\frac{b}{\pi} \int_{-1}^{x_{0}} k(x, t) \ln |1-t| d t+ \\
& +\frac{b}{\pi} \int_{-1}^{x_{0}} k(x, t) \ln \left|x_{0}-t\right| d t-\frac{b}{\pi} \int_{-1}^{x 0} w(t) k(x, t) f_{x_{0}}^{1} \frac{w^{*}(s)-w^{*}(t)}{s-t} d s d t+ \\
& +\frac{b}{\pi} \int_{x_{0}}^{1} k(x, t) \ln \left|x_{0}-t\right| d t-\frac{b}{\pi} \int_{x_{0}}^{1} k(x, t) \ln |1+t| d t+ \\
& +\frac{b}{\pi} \int_{x 0}^{1} w(t) k(x, t) f_{-1}^{x_{0}} \frac{w^{*}(s)-w^{*}(t)}{s-t} d s d t= \\
& =\frac{-b 2^{\kappa}}{\sin \pi \alpha} \int_{x 0}^{1} w(t) k(x, t) P_{\kappa}^{(-\alpha,-\beta)}(t) d t-\frac{b}{\pi} \int_{-1}^{x_{0}} k(x, t) \ln |1-t| d t+ \\
& +\frac{b}{\pi} \int_{-1}^{1} k(x, t) \ln \left|x_{0}-t\right| d t-\frac{b}{\pi} \int_{-1}^{x 0} w(t) k(x, t) f_{x_{0}}^{1} \frac{w^{*}(s)-w^{*}(t)}{s-t} d s d t^{\prime}+
\end{aligned}
$$

53 


$$
\begin{gathered}
-\frac{b}{\pi} \int_{x_{0}}^{1} k(x, t) \ln |1+t|+\frac{b}{\pi} \int_{x 0}^{1} w(t) k(x, t) f_{-1}^{x_{0}} \frac{w^{*}(s)-w^{*}(t)}{s-t} d s d t \\
=\frac{-b 2^{\kappa}}{\sin \pi \alpha} \int_{x 0}^{1} w(t) k(x, t) P_{\kappa}^{(-\alpha,-\beta)}(t) d t+\frac{b}{\pi} \int_{-1}^{1} k(x, t) \ln \left|x_{0}-t\right| d t+ \\
-\frac{b}{\pi} \int_{-1}^{x_{0}} k(x, t)\left[\ln |1-t|+w(t) f_{x_{0}}^{1} \frac{w^{*}(s)-w^{*}(t)}{s-t} d s\right] d t+ \\
+\frac{b}{\pi} \int_{x_{0}}^{1} k(x, t)\left[w(t) f_{-1}^{x_{0}} \frac{w^{*}(s)-w^{*}(t)}{s-t} d s-\ln |1+t|\right] d t
\end{gathered}
$$

Finalmente,

$$
\begin{aligned}
K u(x)= & \frac{-b 2^{\kappa}}{\sin \pi \alpha} \int_{x 0}^{1} w(t) k(x, t) P_{\kappa}^{(-\alpha,-\beta)}(t) d t+\frac{b}{\pi} \int_{-1}^{1} k(x, t) \ln \left|x_{0}-t\right| d t+ \\
& -\frac{b}{\pi} \int_{-1}^{x_{0}} k(x, t) h_{1}(t) d t+\frac{b}{\pi} \int_{x_{0}}^{1} k(x, t) h_{2}(t) d t
\end{aligned}
$$

onde

$$
\begin{aligned}
& h_{1}(t)=\left[\ln |1-t|+w(t) f_{x_{0}}^{1} \frac{w^{*}(s)-w^{*}(t)}{s-t} d s\right] \\
& h_{2}(t)=\left[w(t) f_{-1}^{x_{0}} \frac{w^{*}(s)-w^{*}(t)}{s-t} d s-\ln |1+t|\right]
\end{aligned}
$$

As duas primeiras integrais em ( 5.12), serão aproximadas pela rotina da NAGD01AJF, com tolerância para o erro da ordem de $1.0 E-10$.

Consideremos agora as duas últimas integrais de (5.12). Antes de serem aproximadas pela mesma rotina, devemos fazer um estudo cuidadoso das funções $h_{1}(t)$ e $h_{2}(t)$, dadas respectivamente por (5.13) e (5.14). Estas funções serão calculadas em pontos $t$, fornecidos pela rotina acima mencionada. 


\section{Estudo das funções $h_{1}(t)$ e $h_{2}(t)$}

Para calcular as funções $h_{1}(t)$ e $h_{2}(t)$ devemos observar fundamentalmente a proximidade de $t$, gerado pela rotina da NAG-D01AJF, com $x_{0}$ que é extremo de integração tanto em $h_{1}(t)$ como em $h_{2}(t)$.

Por razões didáticas, o termo proximidade será explicado após a apresentação do Caso 1.

Dois casos podem ocorrer:

Caso 1 : $t$ não está próximo de $x_{0}$.

Neste caso, podemos ter para $h_{1}(t)$ e $h_{2}(t)$ as seguintes expressões

$$
\begin{aligned}
h_{1}(t) & =\ln |1-t|+w(t) f_{x_{0}}^{1} \frac{w^{*}(s)}{s-t} d s-w(t) w^{*}(t) f_{x_{0}}^{1} \frac{d s}{s-t} \\
& =\ln |1-t|+w(t) f_{x_{0}}^{1} \frac{w^{*}(s)}{s-t} d s-\ln |1-t|+\ln \left|x_{0}-t\right| \\
h_{1}(t) & =w(t) f_{x_{0}}^{1} \frac{w^{*}(s)}{s-t} d s+\ln \left|x_{0}-t\right|
\end{aligned}
$$

e

$$
\begin{aligned}
h_{2}(t) & =w(t) f_{-1}^{x_{0}} \frac{w^{*}(s)}{s-t} d s-w(t) w^{*}(t) f_{-1}^{x_{0}} \frac{d s}{s-t}-\ln |1+t| \\
& =w(t) f_{-1}^{x_{0}} \frac{w^{*}(s)}{s-t}-\ln \left|x_{0}-t\right|+\ln |1+t|-\ln |1+t| \\
h_{2}(t) & =w(t) \int_{-1}^{x_{0}} \frac{w^{*}(s)}{s-t} d s-\ln \left|x_{0}-t\right|
\end{aligned}
$$

Usaremos a quadratura de Gerasoulis para as integrais presentes em (5.15) e (5.16), sendo oportuno lembrar aqui, de acordo com o exposto no capítulo anterior, a ineficiência desta quadratura para $t$ próximo de $x_{0}$. Neste caso $h_{1}(t)$ e $h_{2}(t)$ devem ser calculadas pelo $\underline{\text { Caso } 2}$. 
Proximidade A proximidade de $t$ e $x_{0}$ está relacionada com o número de termos $m$ da quadratura de Gerasoulis, calculado pela equação (4.46) para (5.15). Dizemos que $t$ não está próximo de $x_{0}$, se $m<m_{0}$, sendo $m_{0}$ um inteiro positivo previamente escolhido (por exemplo, $\left.m_{0}=100\right)$.

Calculamos de maneira idêntica o número de termos $m$ para (5.16), usando o mesmo valor de $m_{0}$.

Caso 2: $t$ está próximo de $x_{0}$.

Tomando a equação (5.13) podemos determinar $r_{1}$ tal que

$$
h_{1}(t)=\ln |1-t|+w(t) f_{x 0}^{r_{1}} \frac{w^{*}(s)-w^{*}(t)}{s-t} d s+w(t) f_{r_{1}}^{1} \frac{w^{*}(s)-w^{*}(t)}{s-t} d s
$$

Supondo que o $r_{1}$ encontrado não esteja próximo de $x_{0}$ e conseqüentemente de $t$, apenas a primeira integral da expressão anterior requer o uso da quadratura dada por (4.55).

Então, temos para $h_{1}(t)$

$$
\begin{aligned}
h_{1}(t)= & \ln |1-t|+w(t) \sum_{n=1}^{\infty} \frac{A_{n}(x)}{n}\left[\left(r_{1}-t\right)^{n}-\left(x_{0}-t\right)^{n}\right] \\
& +w(t) f_{r_{1}}^{1} \frac{w^{*}(s)}{s-t} d s-f_{r_{1}}^{1} \frac{d s}{s-t} \\
= & \ln |1-t|+w(t) \sum_{n=1}^{\infty} \frac{A_{n}(x)}{n}\left[\left(r_{1}-t\right)^{n}-\left(x_{0}-t\right)^{n}\right] \\
& +w(t) f_{r_{1}}^{1} \frac{w^{*}(s)}{s-t} d s-\ln |1-t|+\ln \left|r_{1}-t\right| .
\end{aligned}
$$

Assim

$$
\begin{aligned}
h_{1}(t)= & w(t) \sum_{n=1}^{\infty} \frac{A_{n}(x)}{n}\left[\left(r_{1}-t\right)^{n}-\left(x_{0}-t\right)^{n}\right] \\
& +w(t) f_{r_{1}}^{1} \frac{w^{*}(s)}{s-t} d s+\ln \left|r_{1}-t\right| .
\end{aligned}
$$

Analogamente, seja $h_{2}(t)$ dada por (5.14).Então

$$
h_{2}(t)=w(t) f_{-1}^{r_{2}} \frac{w^{*}(s)-w^{*}(t)}{s-t} d s+w(t) f_{r_{2}}^{x 0} \frac{w^{*}(s)-w^{*}(t)}{s-t} d s-\ln |1+t|
$$




$$
\begin{aligned}
= & w(t) f_{-1}^{r_{2}} \frac{w^{*}(s)}{s-t} d s-f_{-1}^{r_{2}} \frac{d s}{s-t} \\
& +w(t) \sum_{n=1}^{\infty} \frac{A_{n}(x)}{n}\left[\left(x_{0}-t\right)^{n}-\left(r_{2}-t\right)^{n}\right]-\ln |1+t| \\
= & w(t) f_{-1}^{r_{2}} \frac{w^{*}(s)}{s-t} d s-\ln \left|r_{2}-t\right|+\ln |1+t| \\
& +w(t) \sum_{n=1}^{\infty} \frac{A_{n}(x)}{n}\left[\left(x_{0}-t\right)^{n}-\left(r_{2}-t\right)^{n}\right]-\ln |1+t|
\end{aligned}
$$

Portanto

$$
h_{2}(t)=w(t) f_{-1}^{r_{2}} \frac{w^{*}(s)}{s-t} d s-\ln \left|r_{2}-t\right|+w(t) \sum_{n=1}^{\infty} \frac{A_{n}(x)}{n}\left[\left(x_{0}-t\right)^{n}-\left(r_{2}-t\right)^{n}\right] .
$$

As integrais presentes em (5.17) e (5.18) podem ser aproximadas usando a quadratura de Gerasoulis e $A_{n}(t)$ é dada por (4.54).

Obtenção dos valores de $r_{1}$ e $r_{2}$ : A equação não linear

$$
f\left(\lambda_{2}\right)=0
$$

dada por (4.51), tem uma única solução exata no intervalo $\left[x_{0}, 1\right]$, solução esta que pode ser aproximada, por exemplo, pelo método de bissecção. Usando (4.50), no cálculo de $h_{1}(t)$, o valor de $r_{1}$ é dado por

$$
r_{1}=\frac{\lambda_{2} t-x_{0}}{\lambda_{2}-1}
$$

Importante notar que um raciocínio análogo fornece para $r_{2}$ a expressão dada por (5.19).

\subsubsection{O método de colocação polinomial}

Vamos agora aplicar o método de colocação polinomial à equação (5.6).

De modo análogo ao visto no Capítulo 3 , seja $v_{n}(x)$ a aproximação de $v(x)$, isto é

$$
v_{n}(x)=\sum_{j=0}^{n} a_{j} P_{j}^{(\alpha, \beta)}(x)
$$

onde, como antes, $P_{j}^{(\alpha, \beta)}(x)$ são polinômios de Jacobi de grau $j$ na variável $x$, satisfazendo a relação de recorrência (1.17) e $a_{j}$ são coeficientes a serem determinados. 
Para isto tomemos a equação básica do método de colocação polinomial

$$
r_{n}(x)=\sum_{j=0}^{n} a_{j}\left[\frac{-b 2^{-\kappa}}{\sin \pi \alpha} P_{j}^{(-\alpha,-\beta)}(x)+\int_{-1}^{1} w(t) k(x, t) P_{j}^{(\alpha, \beta)}(t) d t\right]+K u(x)
$$

e o conjunto de pontos de colocação $x_{0}, x_{1}, \ldots, x_{n-\kappa} \in[-1,1]$.

Neste trabalho estamos interessados no caso em que $\kappa=0$, ou seja, na existência de uma única solução .

Usaremos como pontos de colocação as raízes de $T_{n+1}$, dados por (1.18), que apresentam a vantagem adicional de serem independentes da equação integral a ser resolvida.

Impondo que $r_{n}\left(x_{i}\right)=0, i=0,1, \ldots, n$ temos um sistema de equações lineares da forma

$$
A_{i, j} a_{j}=-K u\left(x_{i}\right), \quad i, j=0, \ldots, n
$$

onde

$$
A_{i, j}=\frac{-b 2^{-\kappa}}{\sin \pi \alpha} P_{j}^{(-\alpha,-\beta)}\left(x_{i}\right)+\int_{-1}^{1} w(t) k\left(x_{i}, t\right) P_{j}^{(\alpha, \beta)}(t) d t
$$

Cada $A_{i, j}, i, j=0, \ldots, n$ é determinado usando a quadratura de Gauss dada por (1.22) em (5.23).

Ao sistema (5.22) aplicamos a rotina da NAG-F04ATF, encontrando os valores de $a_{j}$. A solução aproximada $v_{n}(x)$, cuja convergência para $v(x)$ está garantida pelo Teorema da Convergência do Capítulo 3 , é obtida pela substituição dos $a_{j}$ em (5.20).

\subsection{Exemplos Numéricos}

Os exemplos de que tratam esta seção foram resolvidos observando-se os passos descritos abaixo.

1. Dada a equação integral (5.1), cuja solução é $\phi(x)$, devemos em primeiro lugar escolher $w(x)$, de acordo com (2.13). Trabalhamos com $w(x)=(1-x)^{1 / 2}(1+x)^{-1 / 2}$, isto é, $\alpha=1 / 2, \beta=-1 / 2$, consequentemente $\kappa=0$ e a solução é única.

2. Escolha da tolerância $\epsilon$ para o erro admissivel nos cálculos numéricos.

3. Entrada dos valores de $x, x_{0}, a$ e $b$ da equação a ser resolvida. 
4. De acordo com (5.5), cálculo de $u(x)$ pelas equações (5.9) ou (5.10) usando a quadratura de Gerasoulis em ambos os casos, quantas vezes o Algoritmo do Ponto Médio determinar.

5. Cálculo de $K u\left(x_{i}\right), i=0, \ldots, n$ pela equação (5.12), onde $x_{i}$ são os pontos de colocação descritos por (1.18). Para cada $i, h_{1}(t)$ e $h_{2}(t)$ são avaliadas respectivamente por (5.15) e (5.16) usando a quadratura de Gerasoulis ou por (5.17) e (5.18) usando a quadratura para $S_{i}(x)$ e a quadratura de Gerasoulis conjuntamente, de acordo com os casos estudados. É praticamente impossível predizer quantas vezes cada uma destas regras vão ser usadas pois a rotina da Nag-D01AJF gera um número muito grande de valores de $t$ em cada caso (sempre mais que 500 valores para $t$ ).

6. Cálculo dos $A_{i, j}(x)$, para $i=0, \ldots, n$ e $j=0, \ldots, n$ pela equação dada por (5.23) usando a quadratura de Gauss definida em (1.22) para cada $i$ e cada $j$. Assim, a quadratura de Gauss é usada $(n+1)^{2}$ vezes.

7. Cálculo dos $a_{j}$, para $j=0, \ldots, n$ pela resolução do sistema linear de equações de ordem $n+1$ dado por (5.22).

8. Determinação de $v_{n}(x)$ através da equação (5.20).

9. Determinação de $g(x)$ por (5.5).

10. Determinação da solução $\phi(x)$ por $(2.13)$.

Exemplo 1: Consideremos a equação integral

$$
-\frac{1}{\pi} f_{-1}^{1} \frac{\phi(t)}{t-x} d t=f(x)= \begin{cases}0 & x<0 \\ 1 & x>0\end{cases}
$$

que tem como solução exata

$$
\phi(x)=\frac{1}{\pi}\left[\cos ^{-1}\left(x_{0}\right)+\sqrt{\frac{1+x}{1-x}} \log \left|\frac{1+\sqrt{1-x^{2}}}{x}\right|\right] .
$$

A solução numérica desta equação ilimitada em $x=1$ e limitada em $x=-1$ é dada por (2.13), onde $w(x)$ é o definido no início desta seção. Comparando este exemplo
com a equação (5.1), podemos observar que neste caso temos $a=0, b=-1, k(x, t)=0$ e $x_{0}=0$. Escolhemos $\epsilon=1.0 d-10$ e $m_{0}=100$. Para a aplicação do método de colocação usamos

$$
v_{n}(x)=\sum_{j=0}^{16} a_{j} P_{j}^{(1 / 2,-1 / 2)}(x),
$$


e portanto os pontos de colocação são os zeros de $T_{17}(x)$.

É importante ressaltar que neste exemplo, como $k(x, t)=0$, o número de pontos de colocação é irrelevante para obtenção do resultado final.

Na tabela abaixo podemos ver que o método descrito neste trabalho produziu resultados satisfatórios, para diferentes valores de $x$ e até mesmo quando $x$ está bem próximo de $x_{0}=0$ que é a singularidade da solução.

\begin{tabular}{||l|l|l|l||}
\hline$x$ & Solução Aproximada & Solução Exata & Erro \\
\hline 0.5 & 0.7078758528729954 & 0.7078758528737956 & $8.003 \mathrm{E}-13$ \\
\hline 0.2 & 1.137951954279418 & 1.137951954285999 & $6.581 \mathrm{E}-12$ \\
\hline 0.1 & 1.495039440321115 & 1.405039440339856 & $1.874 \mathrm{E}-11$ \\
\hline 0.01 & 2.181523591485810 & 2.181523591722325 & $2.365 \mathrm{E}-10$ \\
\hline 0.001 & 2.918942561285875 & 2.918942566963621 & $5.678 \mathrm{E}-09$ \\
\hline 0.00001 & 4.385308582657443 & 4.385308594566832 & $1.191 \mathrm{E}-8$ \\
\hline-0.01 & 2.191524091594965 & 2.191524091759828 & $1.649 \mathrm{E}-10$ \\
\hline-0.1 & 1.50554322184809 & 1.505543221865777 & $1.727 \mathrm{E}-11$ \\
\hline
\end{tabular}

Tabela 5.1: Solução da Equação integral do exemplo 1.

Exemplo 2: Consideremos agora a seguinte equação

$$
-\frac{1}{\pi} f_{-1}^{1} \frac{\phi(t)}{t-x} d t+\int_{-1}^{1} k(x, t) \phi(t) d t=f(x)= \begin{cases}0 & x<0 \\ 1 & x>0\end{cases}
$$

onde

$$
k(x, t)=\frac{x t}{1+t^{2}+x^{2}}
$$

A solução exata neste caso não é conhecida. Para o cálculo do erro adotamos uma estratégia. Resolvemos a equação de acordo com os passos descritos anteriormente, aplicando o método de colocação para

$$
v_{n}(x)=\sum_{j=0}^{n} a_{j} P_{j}^{(1 / 2,-1 / 2)}(x)
$$

onde $n=50$. Desta maneira foram usados como pontos de colocação os zeros de $T_{51}(x)$. Tomamos esta solução como sendo a exata; fazendo a seguir comparações para valores menores de $n$. 
Observe que, como no exemplo anterior, temos $a=0, b=-1$ e $x_{0}=0$. Escolhemos $\epsilon=1.0 d-10, m_{0}=100$ e $x=0.5$. Aplicando o método para diferentes valores de $n$ os resultados podem ser vistos na tabela abaixo.

\begin{tabular}{||l|l|l||}
\hline \multicolumn{1}{|c|}{$n$} & Solução Aproximada & Erro \\
\hline 5 & -0.9641794283 & $3.322 \mathrm{E}-3$ \\
\hline 10 & -0.9675403676 & $6.127 \mathrm{E}-5$ \\
\hline 15 & -0.9675085930 & $6.953 \mathrm{E}-6$ \\
\hline 20 & -0.9675010609 & $5.793 \mathrm{E}-7$ \\
\hline 25 & -0.9675017103 & $7.006 \mathrm{E}-7$ \\
\hline 30 & -0.9675016339 & $1.215 \mathrm{E}-8$ \\
\hline 35 & -0.9675016576 & $1.730 \mathrm{E}-8$ \\
\hline 40 & -0.9675016465 & $2.761 \mathrm{E}-9$ \\
\hline
\end{tabular}

Tabela 5.2: Solução da Equação integral do Exemplo 2.

\subsection{Conclusão}

A escolha dos zeros do polinômio de Chebyshev de primeira espécie e grau $n+1$ como pontos de colocação se deve aos resultados obtidos em [Cuminato 87], que comprovou a superioridade desta escolha na prática. Entretanto, poderia ter sido usado também os zeros do polinômio de Chebyshev de segunda espécie ou os zeros do polinômio de Jacobi $P_{n+1}^{(1 / 2,-1 / 2)}(x)$.

Os resultados obtidos acima podem ser considerados muito bons, dada a complexidade do problema e do grande número de quadraturas que envolve.

Interessante seria estender os resultados conseguidos, para resolver uma equação integral singular com núcleo de Cauchy e dados não suaves, onde $f(x)$ fosse uma função da seguinte forma:

$$
f(x)=\left\{\begin{array}{ll}
g(x) & x<x_{0} \\
h(x) & x_{0}<x
\end{array} \quad x_{0} \in(-1,1)\right.
$$

Neste trabalho este problema não foi abordado porque não se trata de alguma coisa trivial ou simples generalização, mas requer um estudo cuidadoso e demorado. 


\section{Bibliografia}

[ Abramovitz 64]

[ Bland 70]

[ Boiko 84]

[Cuminato 87]

[Cuminato 90]

[Davis 74]

[Erdogan 71a]

[Erdogan 71b]
ABRAMOVITZ, M., STEGUN, I.A. Handbook of Mathematical Functions. New York, Dover, 1964.

BLAND, S.R. The two-dimensional oscillating airfoil in a wind tunnel in subsonic flow. SIAM J. Appl. Math., v.18, p.830-848, 1970.

BOIKO, A.V.; KARPENKO, L.N. Singular Integral Equations of the plane elasticity problem for a circle with an arbitrary system of cracks and its numerical solution for a branched crack. In: GERASOULIS, A.; VICHNEVETSKY R., eds. Numerical Solution of Singular Integral Equations. New Brunswick, IMACS, 1984.

CUMINATO, J.A. Numerical solutions of Cauchy integral equations and aplications. Tese para obtenção do título de $\mathrm{PhD}$, apresentada ao Wolfson College. Oxford, 1987.

CUMINATO, J.A. Análise de um método de colocação polinomial para solução numérica de equações integrais singulares. Tese para obtenção do título de Livre-Docência apresentada ao ICMSCUSP. São Carlos, 1990.

DAVIS, P.J. Interpolation and Aproximation. New York, Dover, 1974.

ERDOGAN, F.; GUPTA, G. The stress analysis of multi-layered composites with a flaw. Int. J. Solids Structures, v.7, p.39-61, 1971.

ERDOGAN, F.; GUPTA, G. Layered composites with an interface flaw Int. J. Solids Structures, v.7, p.1089-1107, 1971. 
[Erdogan 73]

[Gakhov 66]

[Gerasoulis 86]

[Golberg 79]

[Golberg 84]

[Khvedelidze 56]

[Krenk 75]

[Miller 82]

[Miller 85]

[Monegato 90]

[Muskhelishvili 53]

[Szegö 59]
ERDOGAN, F.; GUPTA, G. Numerical solution of singular integral equations. In: SIH, G.C., ed. Mechanics of Fracture. Groningen, Noordholf, 1973. v.1.

GAKHOV, F.D. Boundary Value Problems. Oxford, Pergamon, 1966.

GERASOULIS, A. Piecewise polynomial quadratures for Cauchy singular integrals. SIAM Num. Anal., v.23, p. 891-902, 1986.

GOLBERG, M.A.; FROMME, J.A. On the $L_{2}$ convergence of a collocation method for the generalized airfoil equation. J. Math. Anal. Appl., v.71, p.271-286, 1979.

GOLBERG, M.A. The convergence of a collocation method for a class of Cauchy singular integral equations. J. Math. Anal.Appl., v. 100, p.500-512, 1984.

KHVEDELIDZE, B.V. Linear discontinuous boundary value problems of the theory of functions, singular integral equations and some applications Trud. Tbilsk. Mat. Inst.; Akad. Nauk. Gruz. $S S R, \quad$ v. 42, p. $3-158,1956$.

KRENK, S. On quadrature formulas for singular integral equations of the first and second kind Q.Appl.Math., v. 33, p. 224-232, 1975.

MILLER, G.F. Numerical treatment of certain singular integral equations arising in problems of accoustic radiation and difraction. In: BAKER, C.T.H.; MILLER, G.F. Treatment of Integral Equations by Numerical Methods. London, Academic Press, 1982.

MILLER, G.F.; KEER, L.M. A numerical technique for the solution of singular integral equations of the second kind. Q.Appl.Math., v. 29 , p. $455-457,1985$.

MONEGATO, G.; LEPORA, P. On the numerical resolution of the generalized air foil equation with Possio kernel. Numer. Math., v.56,p.778, 1990.

MUSKHELISHVILI, N.I. Singular Integral Equation Groningen, Noordhoff, 1953.

SZEGÖ, G. Orthogonal Polynomials. Boston, AMS, 1959. (Colloquium Publication, v.23). 
[Zabreyko 75]

[Wickhan 82]
ZABREYKO, P.P. et al. Integral Equation - A Reference Text. Leyden, Noordhoff, 1975 .

WICKHAN, G.R. Integral equations for boundary value problems exterior to open arcs and surfaces. In: BAKER, C.T.H.; MILLER, G.F. Treatment of Integral Equations by Numerical Methods. London, Academic Press, 1982. 